\title{
Conoscenza e rappresentazione del patrimonio storico costruito in ambiente BIM. Criticità e possibili metodologie applicative
}

Giorgia Potestà

\section{Abstract}

Lo studio indaga le possibili metodologie applicative del Building Information Modeling al patrimonio storico costruito, riflettendo su possibilità e problematiche che il processo può avere in questo specifico campo di applicazione. L'approccio HBIM muove, come per tutti i casi appartenenti al costruito monumentale, da un rilievo integrato, secondo le cosiddette tecniche scan-to-BIM, che consento l'importazione della nuvola di punti all'interno della piattaforma per la modellazione parametrica. Questo processo di ricerca, applicato al battistero di Firenze come caso studio, cerca di definire una metodologia operativa per la modellazione del cultural Heritage fornendo alcuni spunti critici per ricerche future.

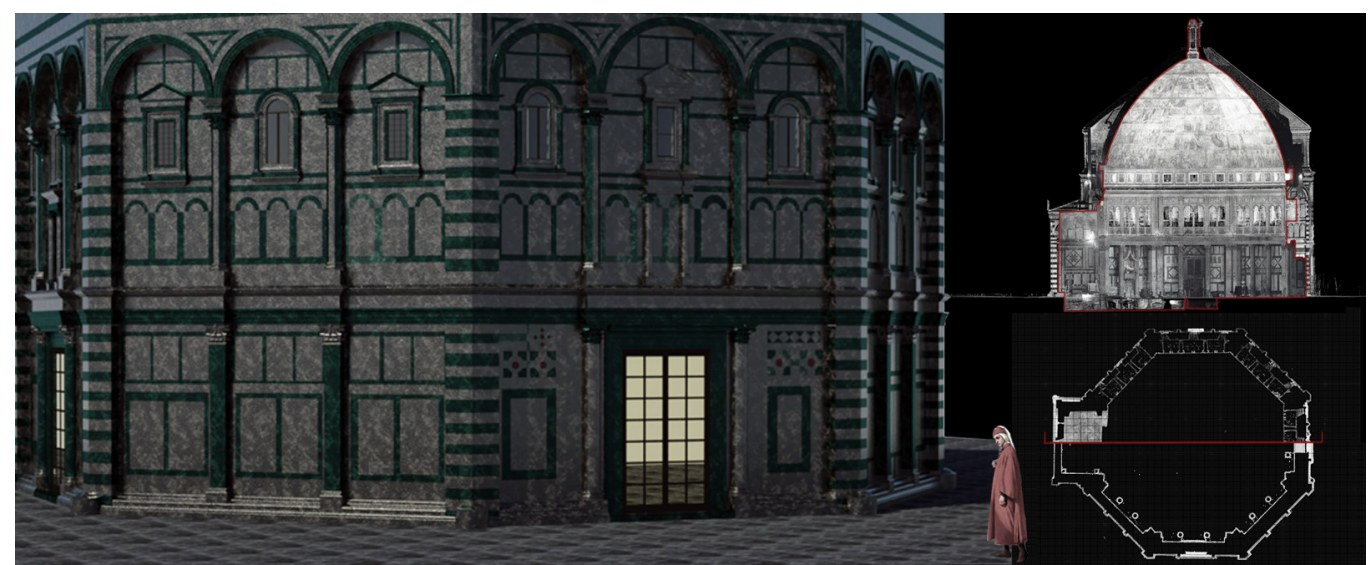




\section{Introduzione}

II potenziale dei sistemi BIM orientati al cosiddetto Heritage-BIM o H-BIM è innegabile; tuttavia rimangono irrisolte le questioni teoriche ed operative relative alla rigidità degli strumenti di modellazione quando sono coinvolti elementi del patrimonio culturale, in cui di fatto si rende necessaria elevata flessibilità.

I modelli 3D per loro natura, sono costituiti da unità geo referenziate, relative in primo luogo alla geometria, topologia, materiali etc. Al contempo le tecnologie di archiviazione digitale, analisi e gestione delle informazioni hanno trovato nei modelli tridimensionali il sostrato su cui sviluppare le proprie potenzialità [Centofanti et al. 20I6]. II BIM offre la possibilità di gestire un'importante quantità di dati eterogenei che, andando oltre il semplice aspetto geometrico, possano costituire un archivio digitale di dati ed informazioni, utile alla documentazione ed alla conservazione dell'architettura storica (fig. I). La traduzione della complessità del costruito storico in un modello tridimensionale digitale pone importanti interrogativi sulle procedure da adottare. La modellazione parametrica e informativa dei monumenti è molto più difficile, sia in termini di trasposizione geometrica della continuità del mondo reale, che della sua descrizione qualitativa e semantica, rispetto alla modellazione ex-novo. L'approccio BIM ad un edificio storico differisce in primo luogo nella quantità e tipologia dei dati di partenza. Nel settore del Cultural Heritage si tratta di una grande quantità di dati a disposizione da dover analizzare e selezionare; occorre stabilire come catalogarli, codificarli ed archiviarli all'interno del modello (o dei modelli) per favorire la massima interoperabilità del dato e per consentire la realizzazione di un modello BIM coerente. Realizzare un buon modello facilita la lettura e la comprensione dell'edificio stesso. II modello BIM del costruito storico differisce anche e soprattutto per le forme architettoniche coinvolte ed il modo di rappresentarle all'interno del modello sotto forma di attributi geometrici ed informativi. Queste difficoltà sono associate soprattutto alla rigidità intrinseca del flusso di lavoro di modellazione parametrica,

Fig. I. Entità coinvolte all'interno del processo BIM per il patrimonio storico costruito. Modello BIM del Battistero di Firenze (elaborazione grafica dell'autore).

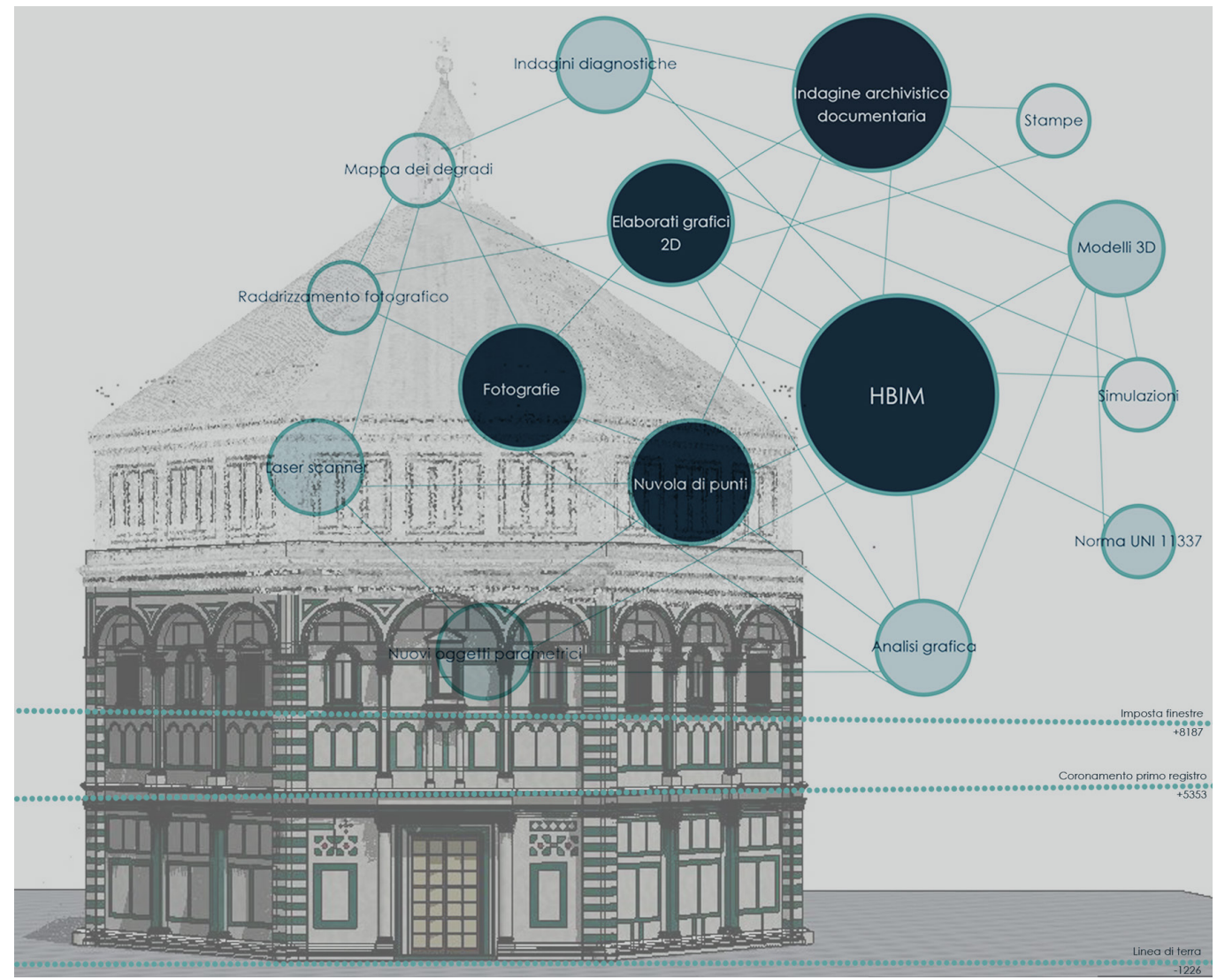


Fig. 2. Segmentazione del Battistero di San

Giovanni. Elaborazione su base ortofoto.

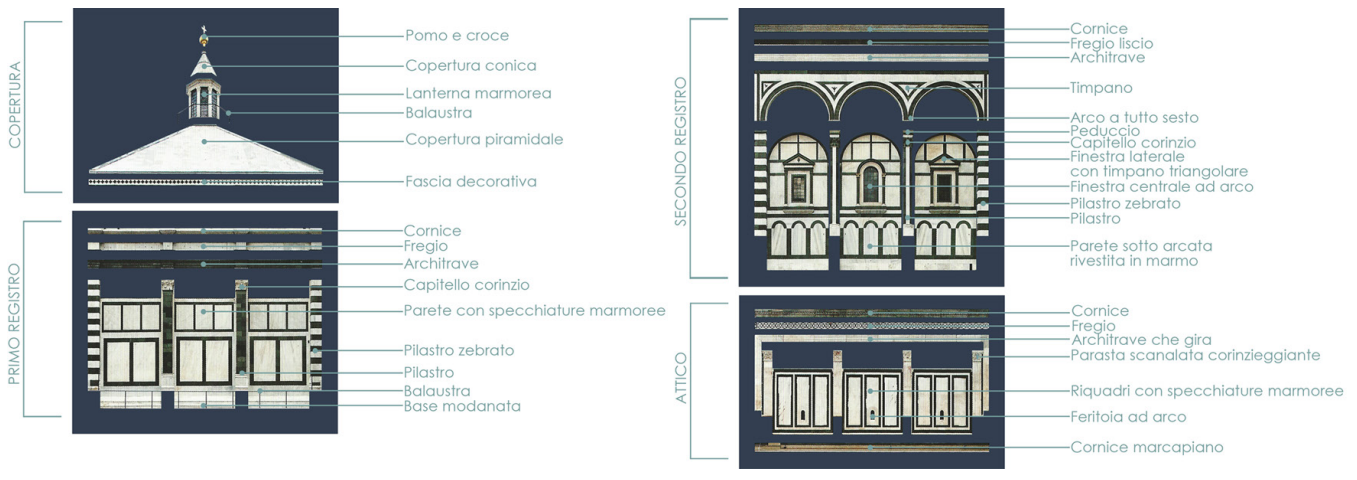

sottoposto a 'librerie' di oggetti digitali che si scontrano con la variabilità e l'unicità dell'ambiente costruito, soprattutto quando 'storico' o in cattivo stato di conservazione. Mentre la transizione da modelli numerici a modelli geometrici implica un'operazione cruciale critica di 'discretizzazione', nella costruzione BIM, il modello comporta un ulteriore passo interpretativo: la strutturazione semantica dei suoi elementi compositivi. Questa operazione ontologica, innegabilmente soggettiva, va ben oltre il riconoscimento delle caratteristiche geometriche degli elementi. Nell'ambiente BIM, infatti, il modellatore procede dal continuum della realtà al digitale discreto esplorando un dominio, additivo e gerarchico, in cui trova agli oggetti digitali il loro posto. L'aggregazione di questi oggetti, sottoposti a rapporti reciproci di incorporazione o appartenenza nonché a vincoli e regole di interazione, guida l'effettiva costruzione del modello informativo virtuale.

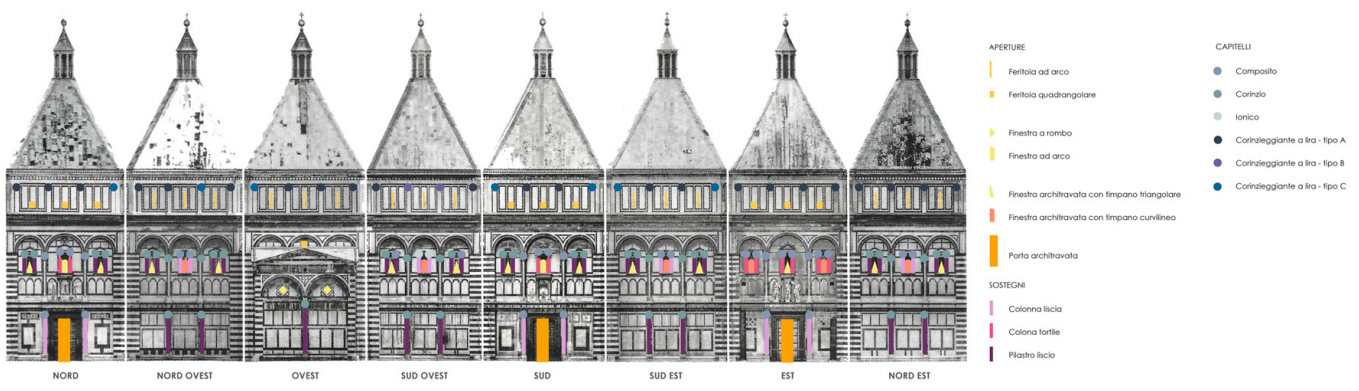

Fig. 3. Individuazione gerarchica degli element architettonici. Elaborazione su base orto foto.

\section{Heritage I Historic BIM}

II termine HBIM è stato coniato da Murphy [Murphy, MC Govern, Pavia 20 I3] che lo presenta come una soluzione innovativa, in cui gli elementi architettonici sono oggetti che integrano diverse informazioni. In quest'ottica, l'HBIM è un sistema interscalare, consistente nella modellazione di elementi architettonici parametrizzati sulla base delle regole dei trattati e dei dati derivanti da indagini sull'edificio [Campi et al. 20 I8]. II sostrato di questi modelli sono le nuvole di punti, da TLS [I] e SfM [2], che interessano l'organismo architettonico storico. Numerose ricerche in questo ambito hanno messo in luce potenzialità e problematiche legate alla definizione di un workflow per la modellazione BIM degli oggetti. Dal plug-in GreenSpider, per la realizzazione di forme [Garagnani 20I3, pp. 489-496], alla definizione di shape grammar specifiche per singole tipologie di elementi costruttivi (es: volte), all'utilizzo di software VPL (Visual Programming Language) come Autodesk Dynamo per la rappresentazione di elementi generabili utilizzando determinate regole e parametri. Si è parlato anche della creazione di famiglie parametriche nidificate, ovvero tramite l'aggregazione gerarchica dei componenti a seconda della funzione strutturale, funzionale e decorativa [Oreni, Bruma- 
Fig. 4. Metodologia di modellazione BIM applicata a patrimonio storico costrulto (elaborazione grafica dell'autore). na, Georgopoulos et al. 20 I3], e, per la modellazione BIM di forme irregolari e complesse, dell'importazione di NURBS a cui vengono aggiunte le informazioni su materiali. Altri ricercatori hanno ragionato sul tema della conversione semi-automatica della nuvola di punti in oggetti semantici [Remondino et al. 20 I8], evidenziando però sempre come ricorrere al processo BIM per il patrimonio costruito risulti piuttosto dispendioso in termini di tempo e risorse impiegate. E importante, quindi, comprendere fino a che Level of Development si debba effettivamente arrivare nella modellazione degli elementi costruttivi base, poiché la creazione di modelli strutturali discontinui in cui vengono discretizzati singolarmente gli elementi della muratura (blocchi o elementi lapidei) e i giunti di malta, è computazionalmente onerosa e difficilmente gestibile [Currà et al. 2019].

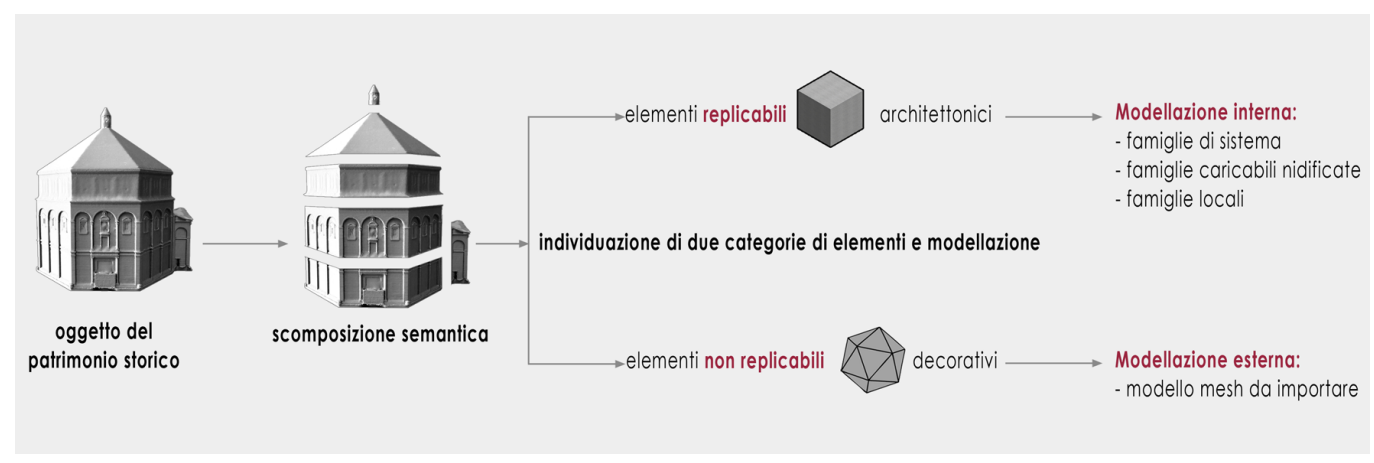

\section{La conoscenza profonda del manufatto}

Lo scopo della ricerca è quello di indagare le modalità di costruzione dei modelli tridimensionali tramite processi HBIM; il primo passo verso la conoscenza del manufatto storico è senza dubbio la raccolta della documentazione storica e la ricerca d'archivio, che possono fornire imprescindibili indicazioni circa la costruzione dell'edificio, le fasi edilizie, le unità di misura usate, i materiali, le stratigrafie murarie etc. I dati acquisiti diventano una prima base di appoggio per la modellazione BIM, alla quale si uniscono i dati di rilievo, che costituiscono un aspetto fondamentale per tutte le attività che riguardano oggetti del patrimonio architettonico esistente. Le nuvole di punti, da TLS alla fotogrammetria digitale che interessano l'organismo architettonico storico offrono una elevata quantità di dati che documenta la condizione esistente degli edifici e descrive una specifica fase del loro ciclo di vita. Non dimentichiamo tuttavia che questi metodi di acquisizione, seppur divenuti lo standard nella pratica del rilievo e della conservazione e gestione del patrimonio culturale, si fermano alla 'pelle' dell'edificio, ignorando la stratigrafia muraria dell'edificio, informazione necessarie al fine di una corretta modellazione BIM, e pertanto da integrare con ulteriori indagini, come termografia, magnetometria etc. o semplicemente con i dati a disposizione dalla documentazione storica, quando reperibili.

\section{Dal rilievo all'HBIM: una metodologia applicativa}

I dati di rilievo costituisco la base di partenza della modellazione, tuttavia occorre articolare il processo metodologico in diverse fasi preliminari alla modellazione. Lo studio di una corretta metodologia applicativa facilita la modellazione BIM e di fatto se ne rende necessaria soprattutto nel campo del Cultural Heritage. In questo specifico campo applicativo occorre più che mai partire, dalla scomposizione dell'edificio in elementi costitutivi; ogni manufatto può essere scomposto in un insieme coordinato di elementi semplici e complessi, che ne determinano assieme la struttura. L'analisi e la scomposizione di queste parti all'interno di un'architettura costruita viene definita, in maniera più o meno appropriata, segmentazione 
semantica. L'operazione di segmentazione facilita lo studio dei monumenti storici ed integra informazioni ed attributi eterogenei, utili a descrivere e caratterizzare l'oggetto in questione. L'edificio può essere descritto come una raccolta di elementi organizzati gerarchicamente, identificati da un vocabolario architettonico specifico [Fallavolita et al. 20 I 8]. Un'attenzione particolare in questa operazione va posta nell'analizzare come questi oggetti sono assemblati da un punto di vista tipologico e morfologico poiché questa problematica si presenta ogni volta che si approccia alla modellazione BIM di un manufatto: comprendere come ogni elemento si costituisce e come si lega agli altri all'interno del modello.

La segmentazione degli edifici storici richiede inoltre un vocabolario architettonico correlato dalla conoscenza di tutte quelle componenti strutturali, funzionali e decorative, come modanature, ordini architettonici, trabeazioni e profili, e delle loro caratteristiche geometriche e topologiche, in termini di caratteristiche e connessioni tra le parti, al fine di descrivere completamente l'edificio (figg. 2, 3). La grande quantità di dati e di modelli digitali prodotti fa emergere la necessità di catalogare e classificare i dati ottenuti attraverso strumenti di supporto della fase interpretativa. Nuove procedure e soluzioni di modellazione e annotazione semantica rendono più agevoli e speditive le operazioni di segmentazione, secondo approcci diversi come color similarity e spacial proximities, shape detection, distance between planar faces [Hichri et al. 2013].

Come già detto, quando ci si approccia ad un modello BIM per il costruito storico, particolare attenzione va posta alle forme architettoniche coinvolte ed al modo di rappresentarle all'interno del modello sotto forma di attributi geometrici. Occorre infatti prendere le distanze dalla metodologia operativa propria dei modellatori tradizionali, che permette la realizzazione di geometrie complesse con operazioni di loft, sweep e estrusioni particolari. Al contrario è necessario, dopo aver definito quali sono gli elementi da modellare e come sono relazionati tra loro, definire i metodi di modellazione anche in funzione delle informazioni che si hanno a disposizione. Si sono riconosciute due macro categorie di elementi da modellare: elementi replicabili ed elementi unici non ripetibili. Allinterno della prima

Fig. 5. Metodologie monumento: terrestria laser scannig e immagini gigapixel (in alto). Rilievi desunti dalla ricerca storico archivistica: pianta e alzata e taglio del Tempio di San Gio. Batista, incisione di Bernardo Sgrilli su disegno di Giovan Battista Nelli in Descrizione e Studi dell'insigne Fabbrica di S. Maria del Fiore, Firnze 1733, tav XVII, e Disegno del Corinti, scavi del 1895 in Pietramellara C. Battistero di San Giovanni a Firenze, rilievo e studio critico, 1973.
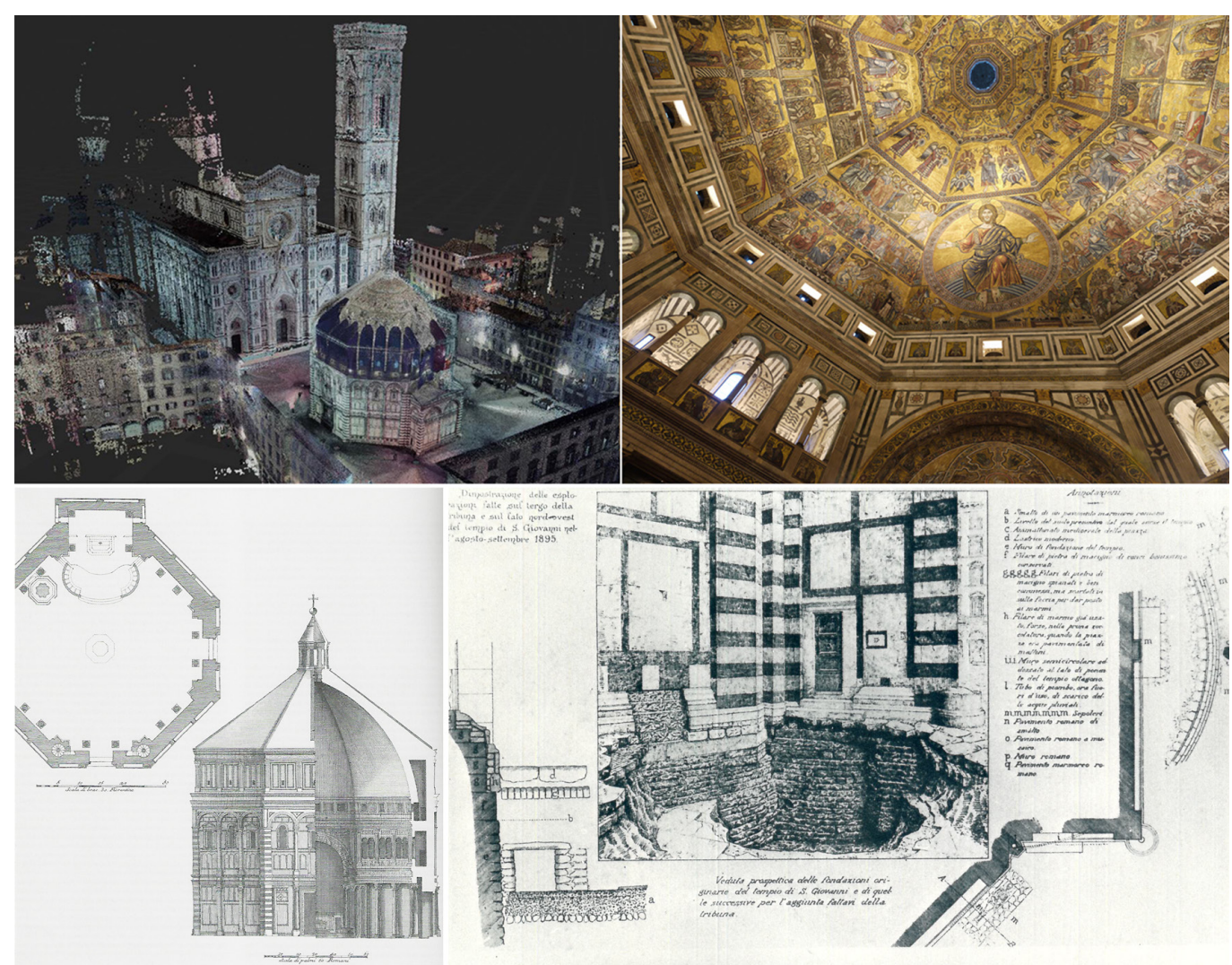
Fig. 6. Impostazione delle scanregions dentro Autodesk Recap e visualizzazione delle stesse all'interno di Revit.

Fig. 7. Modellazione della massa dalla nuvola di punti e applicazione della famiglia di sistema muro.

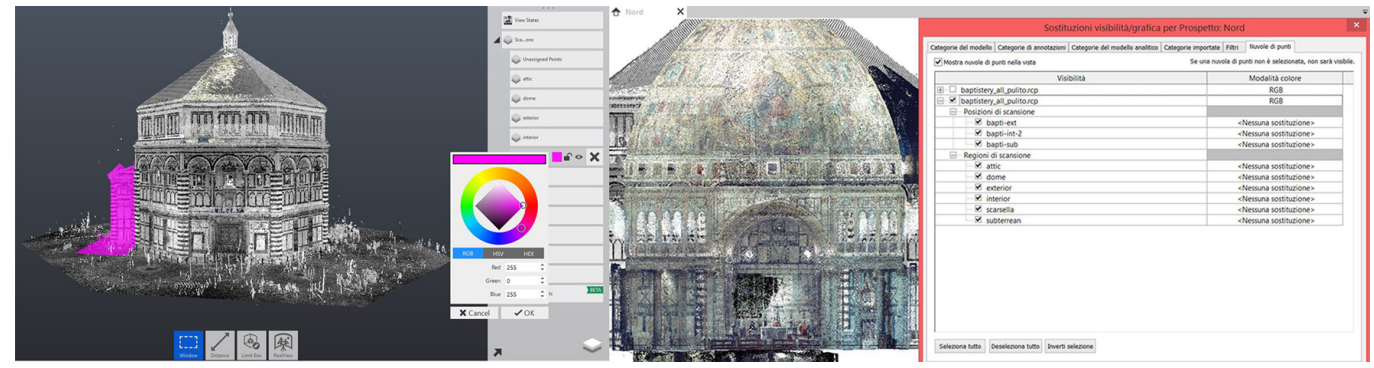

categoria rientrano gli elementi architettonici e costruttivi, come tipologie di muri, pavimenti, volte, colonne, capitelli, pilastri, finestre e trabeazioni; nella seconda rientrano gli elementi sculterei e lapidei, suppellettili e tutti quegli elementi che costituiscono un unicum all'interno dell'edificio. Una volta inquadrate le distinzioni tra categorie di elementi, si andrà ad analizzare quali sono i diversi metodi di modellazione degli stessi. Gli elementi replicabili sono stati modellati tutti all'interno della piattaforma BIM, utilizzando famiglie di sistema per quanto riguarda gli elementi di chiusura orizzontale e verticale come muri, pavimenti e copertura, e famiglie caricabili nidificate o modellazione locale per tutti gli elementi architettonici. All'interno del software si ha la possibilità, per le parti strutturali, di modellarle direttamente tramite specifici comandi di estrusione oppure, soluzione conveniente quando si hanno geometrie meno regolari come nel caso del patrimonio storico, attraverso la costruzione di una massa alla quale verranno applicate superfici murarie e solai individuando gli opportuni piani di riferimento.

Per ciò che riguarda invece la seconda categoria di oggetti, elementi non replicabili caratterizzati da geometrie complesse, come gli elementi scultorei sopra le porte o le suppellettili interne, si procede tramite realizzazione di modelli mesh ad hoc su nuvola di punti oppure modelli di SfM; in entrambi i casi i modelli ottenuti dovranno essere importati successivamente nella piattaforma BIM (fig. 4). Questa operazione di importazione tuttavia non sarà facile poiché la piattaforma BIM utilizzata consente l'importazione solo di formati CAD, e sarà necessario ricorrere a più di un passaggio.

\section{Applicazione al caso studio: II Battistero di San Giovanni a Firenze}

|| Battistero fiorentino, o “ll Bel San Giovanni” come lo chiamava Dante Alighieri [3], è una costruzione in muratura a pianta ottagonale piuttosto regolare, interrotta sul lato sud dall'abside quadrangolare, detto scarsella. Da un punto di vista strutturale si riconoscono in altezza due livelli: il primo, dalla quota del pavimento all'intradosso del matroneo, e l'altro
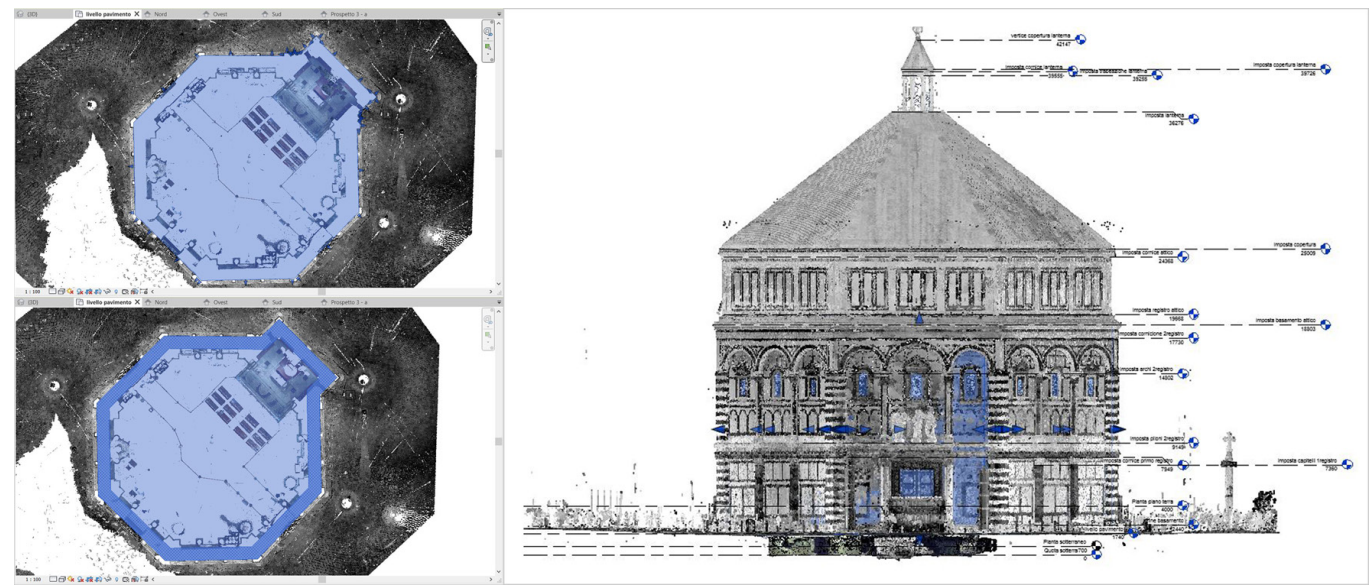
dall'estradosso del matroneo fino alla galleria [Bartoli et al. 20 I7]. L'analisi delle fasi costruttive del monumento è propedeutica alla comprensione di questo, nonché alla possibilità di inserire queste informazioni all'interno della piattaforma BIM per ogni elemento modellato attraverso l'uso dei 'filtri fase'. Sono state riconosciute tre fasi principali per la costruzione del manufatto: una prima fase, alla fine del XI secolo, con la costruzione del grezzo di forma ottagona, una seconda fase, datata tra la fine del XI secolo ed i primi del XII, con la realizzazione del rivestimento marmoreo dei primi due registri esterni e la costruzione della lanterna, ed un'ultima fase, datata alla prima metà del XIII secolo, che vede il rivestimento integrale delle superfici di copertura e dei matronei con il mosaico a fondo oro e la realizzazione della scarsella [Paolucci 1994].

La campagna di acquisizione è stata fatta integrando diverse metodologie non a contatto: TLS, SFM, indagini termografiche infrarossi, acquisizione di immagini gigapixel (fig. 5). I dati sono stati integrati e gestiti all'interno del software di point cloud rendering Viscore di CISA3 [4]. Sono state eseguite 62 scansioni totali, allineate in una nuvola di punti complessiva con più di mezzo bilione di punti 3D acquisiti. Le nuvole di punti acquisite da laser scanner Faro sono state registrate ed allineate all'interno del software Viscore, dal quale è stato possibile ottenere una serie di shapshoots ad alta risoluzione prepedeutici alla modellazione degli elementi di dettaglio; da questo si è passati poi all'interno di Autodesk Recap per favorire l'importazione della nuvola in Revit. Operazione fondamentale all'interno di Recap è stata quella di raggruppare la scansioni, dividendole per acquisizione dell'esterno, dell'interno e dei sotterranei, e soprattutto la creazione di scanregions (fig. 6) specifiche per la cupola, i diversi registri e la scarsella, in modo tale da facilitare la modellazione diretta dalla nuvola in Revit, in quanto la piattaforma BIM nella fase di importazione della nuvola, permette anche l'importazione delle eventuali segmentazioni effettuate. Una volta importata la nuvola di punti si è proceduto con la geolocalizzazione del progetto e la definizione dell'orientamento, allineando il nord di progetto al nord reale e generando pertanto viste e prospetti in linea con le definizioni utilizzate fin oggi. Si è poi proceduto con l'impostazione di piani di riferimento, essenziali non solo per generare viste in pianta o sezione, ma soprattutto per vincolare gli oggetti modellati a specifici piani o allineamenti dell'architettura stessa. Si è scelto di iniziare a modellare l'involucro dell'edificio come massa, come già anticipato, direttamente rilucidando dalla nuvola di punti importata (fig. 7), e la modellazione è avvenuta per fasi edi-

Fig. 8. Modellazione parametrica della famiglia nidificata colonna ionica tramite famiglie profilo per base ed abaco e famiglia modello generico metrico per le volute.

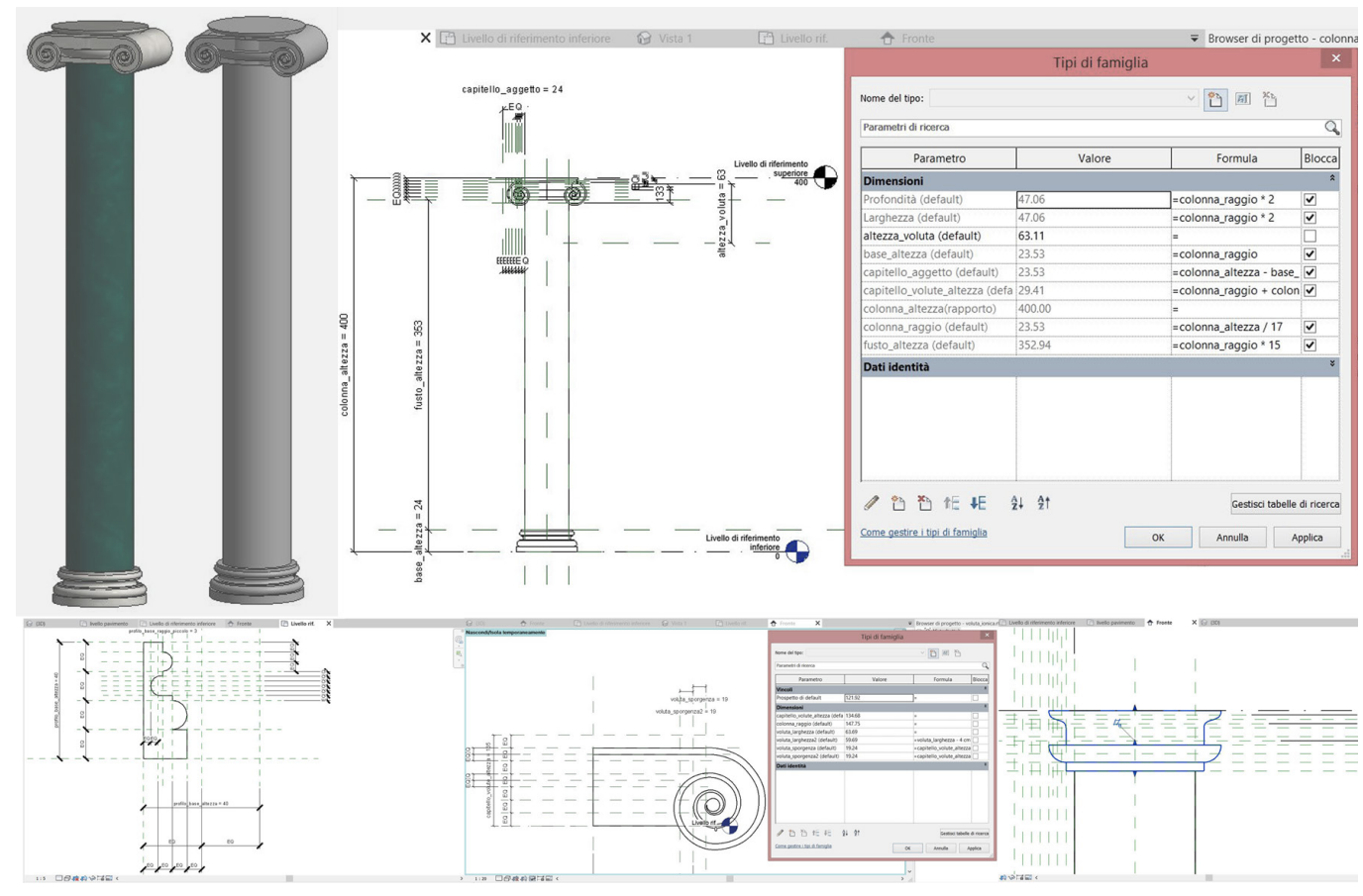


lizie successive, procedendo per registri. In questa fase tutto viene modellato direttamente sulla base della nuvola di punti. La stratigrafia muraria è stata definita sulla base dei recenti studi e analisi strutturali che hanno riguardato il monumento e che si sono rintracciate in bibliografia [5]. E' stato applicato uno strato di finitura esterna in marmo di spessore $8 \mathrm{~cm}$ e successivamente si sono andate a definire le singole lastre di rivestimento applicando le finiture in marmo bianco Carrara,Verde di Prato e Rosso Cintoia. Tutti gli elementi architettonici ripetibili, come porte, finestre, colonne e dadi trabeati, sono stati trattati come famiglie caricabili nidificate, owero come elementi modellati separatamente nella piattaforma BIM e che vengono successivamente caricati all'interno del progetto (fig. 8). Si tratta di famiglie nidificate più o meno complesse; da quelle in cui viene utilizzata una famiglia profilo per ottenere un'estrusione su binario, a quelle in cui vengono importate sia famiglie profilo che famiglie di modelli generici metrici, per esempio per la realizzazione delle colonne o delle finestre (fig. 9). I profili sono stati modellati o direttamente in Revit in maniera parametrica o su base CAD e poi importati. Altri elementi come cornici modanate e trabeazioni che

Fig. 9. Modellazione parametrica in pianta e alzato della famiglia nidificata finestra timpanata tramite famiglie profilo per le estrusion delle trabeazioni, del timpano e del davanzale e importazione della famiglia nidificata parasta composita (a destra). La famiglia finestra caricata e posizionata del file di progetto (a sinistra)

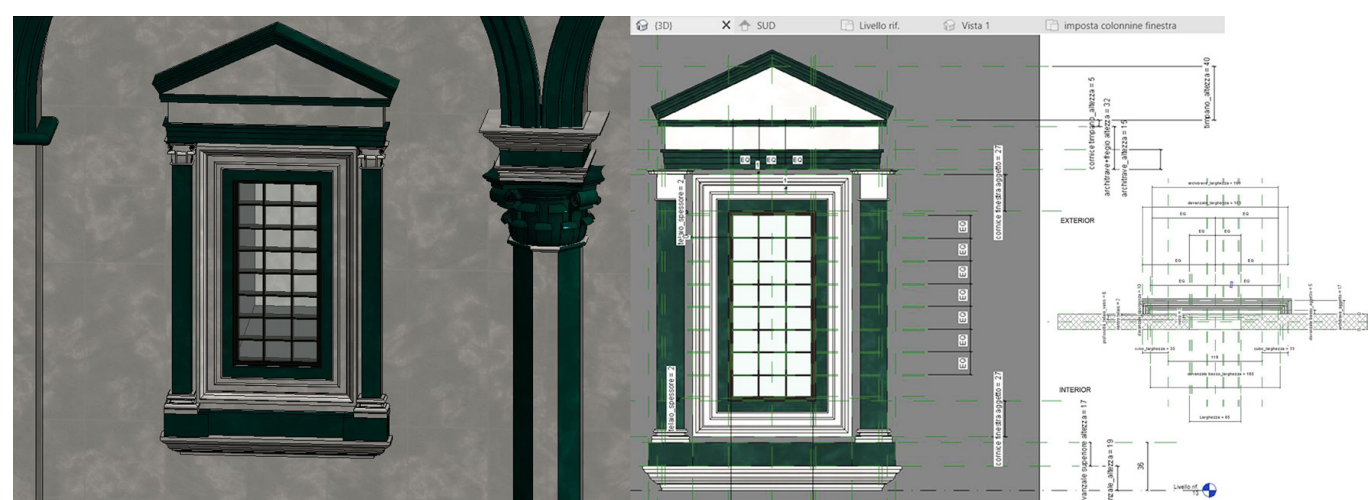

corrono lungo tutto l'edificio sono state modellate come componente locale importando il profilo da estrudere direttamente nel progetto e applicando in place il materiale costruttivo. II punto dolente in questo tipo di modellazione non è altro che il tempo impiegato per ottenere un determinato modello; ai fini pratici infatti è possibile modellare qualunque tipo di elemento architettonico nella piattaforma, bisogna tuttavia scomporlo nelle sue diverse parti e modellarle come ulteriori famiglie parametriche. La modellazione parametrica non è altro che il riconoscimento delle singole parti che compongono l'elemento architettonico e del rapporto proporzionale di dipendenza reciproca di una dall'altra parte; modellare in modo parametrico equivale ad applicare le 'regole', per moduli o per partizioni successive, alla modellazione dell'architettura.

Come la maggior parte dei software di modellazione parametrica, anche Revit non è in grado di estrarre dei dati dai punti di interesse della nuvola in modo da garantire una fedele modellazione delle geometrie, ma come accade per la restituzione 2D, anche nella modella-

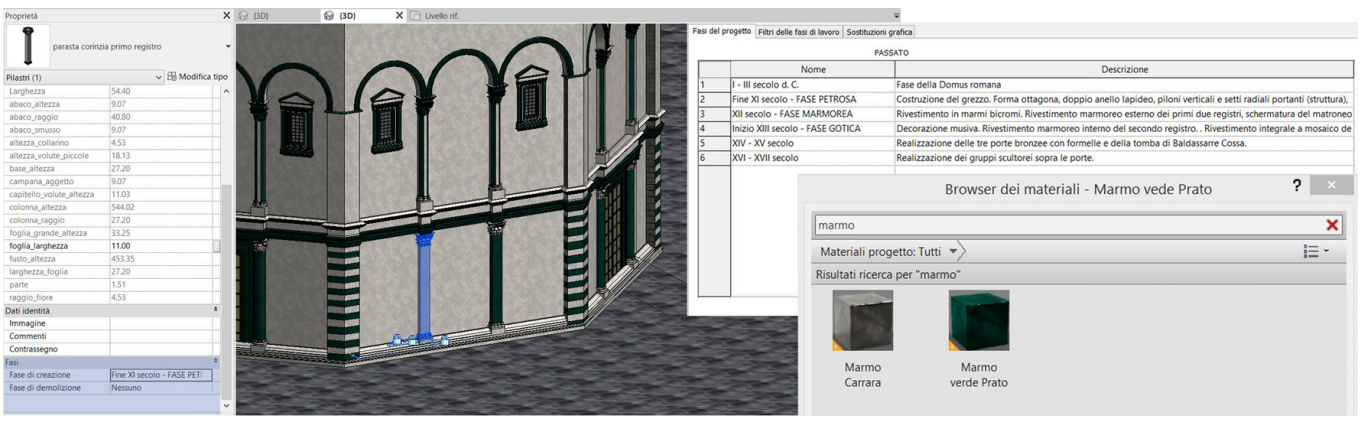

Fig. I0. Applicazione del filtro fase all'elemento parasta corinzia del primo registro (a sinistra). Browser delle fasi edilizie inserite e dei materiali utilizzati per l'elemento parasta (a destra). 
Fig. II. Ridefinizione del data flow del processo BIM attraverso i LOD e rappresentazione dei livelli tematici. LOd di un podio (elaborazione grafica dell'autore): LOR del tempio del Divo Claudio Attenni 20197: LE della [Attenni 2019], LOE della [Bianchini, Nicastro 2018] zione BIM la nuvola di punti viene trattata come una base di dati, un'immagine da rilucidare e da cui ottenere la geometria dell'edificio, e impiegata come traccia metrica. Questo processo viene chiaramente eseguito manualmente e di conseguenza implica un forte grado di soggettività nell'interpretazione e nell'esecuzione delle operazioni di modellazione.

Si è infine indagato un ulteriore aspetto del modello, ovvero quello della possibilità di inserire filtri di costruzione degli elementi, i cosiddetti filtri fase. Questi consentono, nella fase di modellazione e gestione dell'elemento modellato, di inserire informazioni circa la fase di creazione dell'elemento, relazionandola quindi all'epoca di creazione (fig. I0). I filtri fase danno perciò la possibilità di generare un modello architettonico che raccolga in se anche un'analisi sincronica del manufatto, ovvero che nel suo stato as is dia conto della datazione di alcune sue parti, visualizzandole contemporaneamente. Ovviamente la piattaforma dà anche la possibilità, cosi come per la modellazione di nuovi edifici, di generare differenti viste per ciascun filtro fase, dando perciò la possibilità di avere un'analisi diacronica, ovvero di visualizzare separatamente gli elementi che appartengono distintamente a ciascuna fase edilizia.

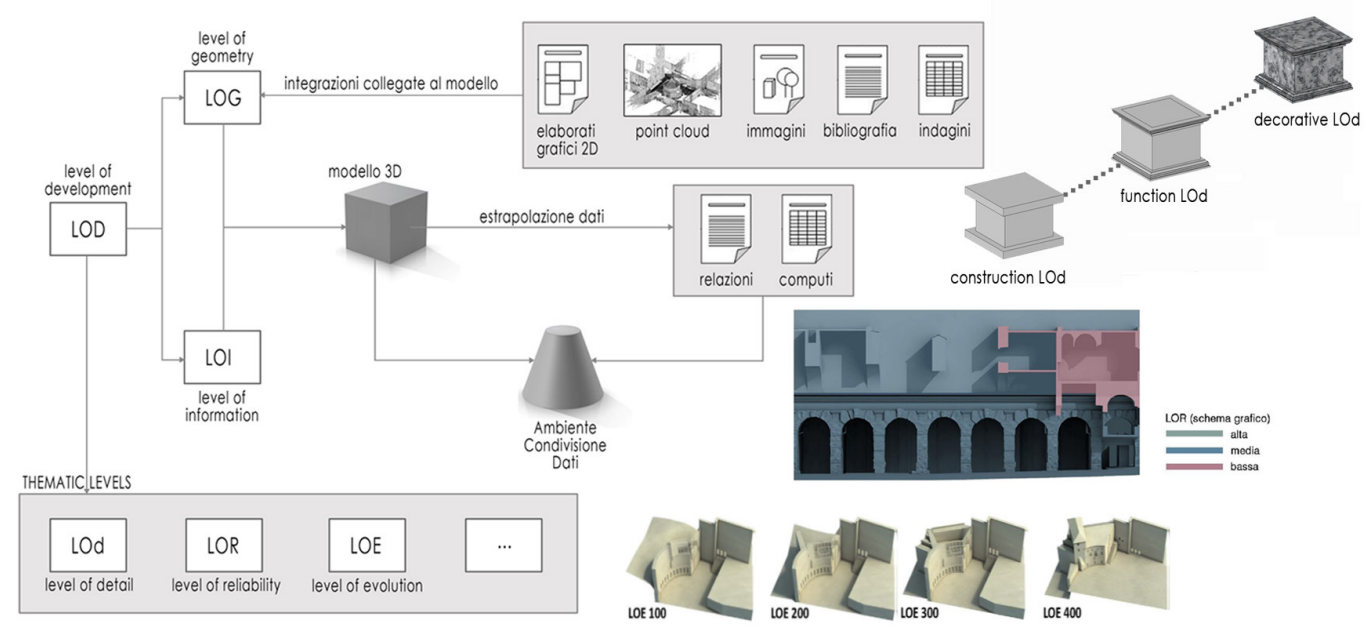

\section{Conclusioni}

La ricerca ha cercato di indagare i diversi aspetti della modellazione BIM di un manufatto storico, cercando di definire un possibile processo operativo ed evidenziandone alcuni limiti. Quando si approccia alla modellazione BIM di un manufatto storico, occorre in primo luogo chiarire quale siano gli obiettivi e soprattutto l'utilizzo di tale modello, in modo da definire in maniera preventiva anche i livelli di dettaglio dello stesso [Rossi, Palmieri 20l8]. Risulta evidente inoltre che i LODs non saranno mai omogenei all'interno dello stesso, in quanto inficiati dalla reale conoscenza dei singoli elementi modellati; a tale fine bisognerebbe sempre rendere evidente, per ogni modello, il Level of Reliability degli elementi [Bianchini, Nicastro 2018], in modo tale da avere sempre un controllo sulle parti e consentirne gli aggiornamenti. Le diverse rappresentazioni che possiamo fare all'interno di un modello BIM dei LOR o dei diversi Levels of Detail o delle fasi edilizie a cui ogni elemento appartiene (Levels of Evolution), sono essi stessi dei modelli, definiti come 'modelli tematici' (fig. I I), scelti in funzione di specifici obiettivi.

II crescente utilizzo di questi mezzi di rappresentazione per i progetti riguardanti il campo dell'architettura, dell'ingegneria e ora anche del restauro, ci impone, come studiosi, di trovare metodologie operative per l'applicazione in campo culturale senza dimenticare il fine che tale modellazione si pone. Se si guarda oltre il primo scoglio, ovvero quello per cui la modellazione BIM nasce per le nuove costruzioni, per elementi standardizzati, e si cerca di 
definire una strategia operativa per applicarlo al campo dei beni culturali, ci si accorge che la modellazione parametrica non è altro che la rappresentazione dell'architettura come l'abbiamo sempre riconosciuta, ovvero secondo il concetto vitruviano di partizioni successive e di Ordine Architettonico, e che la possibilità di allegare al modello un'enorme quantità di informazioni circa materiali, caratteristiche e tutte quello che vogliamo ricomprendere all'interno delle schede di proprietà, si pone come una sensazionale opportunità per il campo del restauro, della gestione e della rappresentazione dell'architettura storica. II BIM ci offre, come studiosi nel campo del patrimonio culturale, l'opportunità di raccogliere per la prima volta tutte le informazioni in nostro possesso all'interno di un'unica piattaforma (fig. 12) nella quale è possibile anche e soprattutto modellare l'oggetto. Allora perché escludere a priori questo tipo di rappresentazione tridimensionale e preferirne un'altra? Ad ogni modo, ogni volta che ci si approccia alla modellazione è sempre bene aver chiari gli obiettivi che tale progetto si pone.

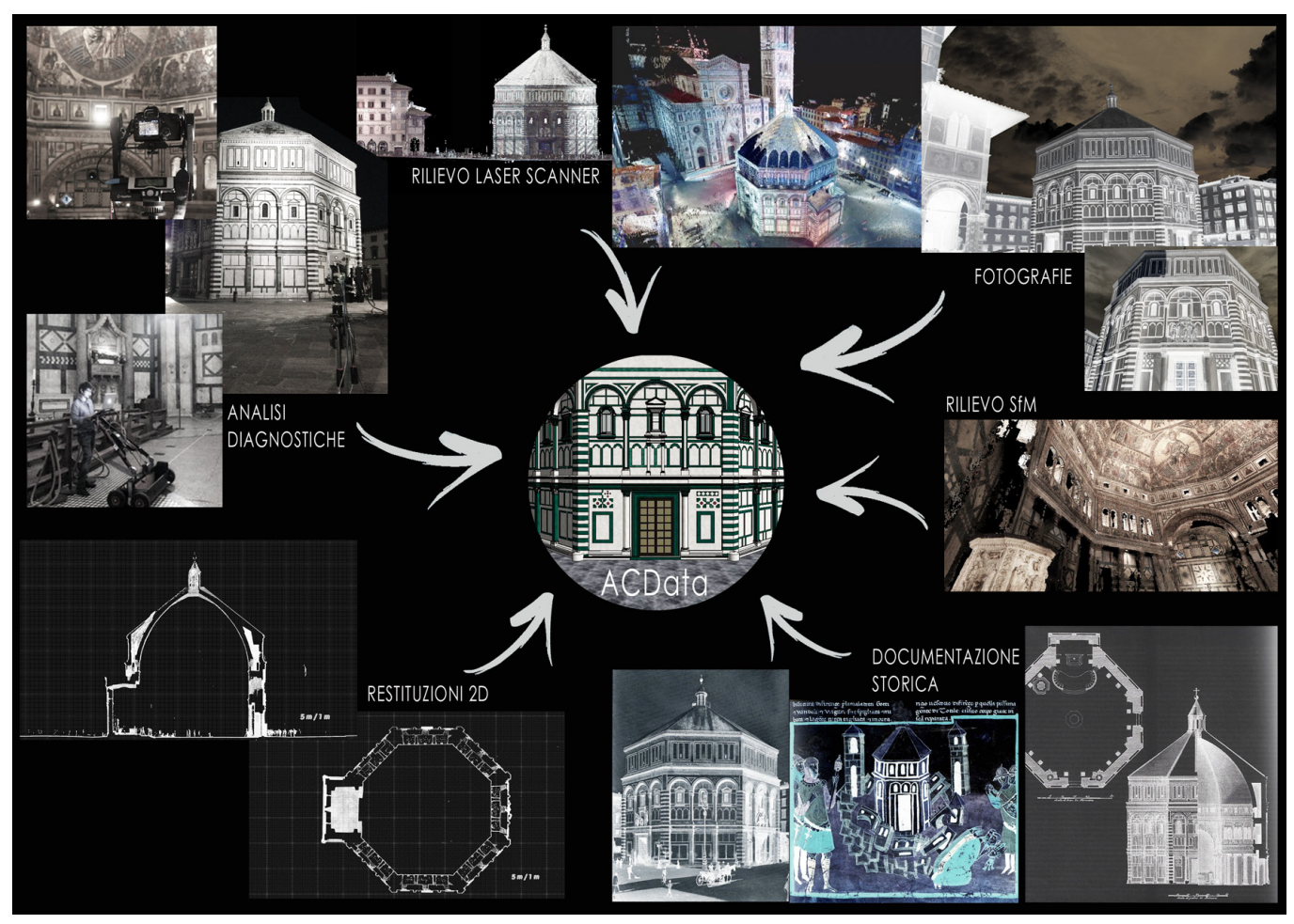

Note

[I]Terrestrial Laser Scanner.

[2] Structure from Motion.

[3] "Non mi parean né ampi né maggiori che que' che son nel mio bel S. Giovanni, fatti per luogo di battezzatori" Dante Alighieri, Divina Commedia, Inferno, XIX, 16-18.

[4] Center of Interdisciplinary Science for Art, Architecture, and Archaeology della University of California San Diego.

[5] L'intero spessore murario è caratterizzato da uno strato di finitura interno ed esterno in lastre di marmo di spessore $8 \mathrm{~cm}$; il nucleo interno è caratterizzato da una muratura compatta in cui lo spessore totale è $180 \mathrm{~cm}$ suddiviso in tre strati. 


\section{Riferimenti bibliografici}

Attenni Martina (2019). L'architettura romana al tempo del BIM. In Empler Tommaso, Fusinetti Alexandra (a cura di). 3D Modeling \& BIM. Modelli e soluzioni per la digitalizzazione. Roma: dei.

Bartoli Gianni, Betti Michele, Monchetti Silvia (2017). Modellazione numerica ed analisi strutturale del Battistero di San Giovanni a Firenze. In Gurrieri Francesco. II battistero di San Giovanni: conoscenza, diagnostica, conservazione. Atti del convegno internazionale Firenze, 24-25 novembre 20 I 4. Firenze: Mandragora, pp. I35- 157.

Bianchini Carlo, Nicastro Saverio (2018). La definizione del Level of Reliability: un contributo alla trasparenza dei processi historic-BIM. In Empler Tommaso, Fusinetti Alexandra (a cura di). 3D modeling \& BIM, applicazioni e possibili futuri sviluppi. Roma: dei, pp. 209-225

Bianchini Carlo, Nicastro Saverio (20 18). From BIM to HBIM. In 2018 3rd Digital Heritage International Congress (Digital Heritage) held jointly with 2018 24th International Conference on Virtual Systems \& Multimedia (VSMM 2018).

Campi Massimiliano, Cera Valeria, Farella Elisa et al. (2018). Un catalogo semantico per la conoscenza e la ricostruzione del paesaggio incompiuto. Il caso di San Leucio. In Capano Francesca, Pascariello Maria Ines,Visone Massimo (a cura di). Delli aspetti de paesi. Vecchi e nuovi media per l'immagine del paesaggio. Napoli: FedOA, vol. 2, pp. 229-237.

Carnevali Laura, Lanfranchi Fabio, Russo Michele (2019). Built Information Modeling for the 3D Reconstruction of Modern Railway Stations. In Heritage an Open Access Journal by MDPI (2), pp. 2298-23I0.

Centofanti Mario, Brusaporci Stefano, Maiezza Pamela (2016). Tra "HistoricalBIM" ed "HeritageBIM": Building Information Modeling per la documentazione dei beni architettonici. In Parrinello Sandro, Besana Daniela. Reuso 20I6. IV Convegno Internazionale sulla documentazione, conservazione e recupero del patrimonio architettonico e per la tutela paesaggistica. Firenze 6-8 ottobre 20 |6. Firenze: Edifir, pp. 42-51.

Currà Edoardo, D'amico Alessandro, Angelosanti Marco (2019). HBIM per la conoscenza e la rappresentazione della costruzione storica. II caso di villa Palma - Guazzaroni a Terni. In Garda Emilia, Mele Caterina, Piantanida Paolo. Colloqui. AT. e Ingegno e costruzione nell'epoca della complessità. Atti del congresso Torino, 25-27 settembre 20 I 9. Torino: Politecnico di Torino, pp. 628-637.

Fallavolita Federico, Ballabeni Massimo, Foschi Riccardo, et al (20 I5). Semantic description of the three-dimensional models of Bologna porches. In SCIRES.IT, Vol 5, Issue I, pp. 3I-40.

Garagnani Simone (20/3). Building Information Modeling and real world knowledge: A methodological approach to accurate semantic documentation for the built environment. In Digit 20 / 3. Fed I9th Int'IVSMM, I Oth Eurographics GCH, 2nd UNESCO Mem World Conf Plus Spec Sess fromCAA, pp. 489-496.

Hichri Nourredine, Stefani Chiara, De Luca Livio, et al (2013). Review of the "as-built BIM" approaches. In The International Archives of the Photogrammetry, Remote Sensing and Spatial Information Sciences. Proceedings of the 3DARCH 20I3. Trento, 25-26 February 20 I 3. Göttingen: Copernicus GmbH, vol. XL-5/WI, pp. I07- I I 2

Murphy Maurice, MC Govern Eugene, Pavia Sara (2013). Historic Building Information Modelling - Adding intelligence to laser and image based surveys of European classical architecture. In ISPRS Journal of Photogrammetry and Remote Sensing, n. 76, pp. 89- 102

Oreni Daniela, Brumana Raffaella, Georgopoulos Andrea et al. (2013). Hbim For Conservation And Management Of Built Heritage:Towards A Library Of Vaults And Wooden Bean Floors. In ISPRS Annals of the Photogrammetry, Remote Sensing and Spatial Information Sciences, Volume II-5/WI, 20I 3 -XXIV International CIPA Symposium, 2-6 September 20 I 3, Strasbourg, Pp. 215-22|.

Paolucci Antonio (1994). Il battistero di San Giovanni a Firenze. Firenze: Cosimo Panini Editore.

Remondino Fabio, Grilli Eleonora, Petrucci Giulio (2018). Supervised segmentation of 3D cultural heritage. San Francisco: IEEE.

Rossi Adriana, Palmieri Umberto (2019). LOD per il patrimonio architettonico: la modellazione BIM per la fabbrica di Solimene. In Disègno, n. 4, Giugno 2019

Autore

Giorgia Potestà, La Sapienza Università di Roma, giorgia.potesta@uniromal.it

Per citare questo capitolo: Potestà Giorgia (2020). Conoscenza e rappresentazione del patrimonio storico costruito in ambiente BIM. Criticità e possibili metodologie applicative/Knowledge and representation of Cultural Heritage in a BIM environment. Critical issues and possible application methodologies. In Arena A., Arena M., Brandolino R.G., Colistra D., Ginex G., Mediati D., Nucifora S., Raffa P. (a cura di). Connettere. Un disegno per annodare e tessere. Atti del $42^{\circ}$ Convegno Internazionale dei Docenti delle Discipline della Rappresentazione/Connecting. Drawing for weaving relationships. Proceedings of the 42th International Conference of Representation Disciplines Teachers. Milano: FrancoAngeli, pp. I 363 - I 384. 


\section{Knowledge and Representation of Cultural Heritage in a BIM Environment. Critical Issues and Possible Application Methodologies}

Giorgia Potestà

Abstract

The research investigates the possible application methodologies of Building Information Modeling to the historical built heritage, thinking on the possibilities and problems that the process may have in this specific field of application. The HBIM approach moves, as for all cases belonging to the historical building, from an integrated survey, according to the so-called scan-to-BIM techniques, which allow the import of the point cloud inside the parametric modeling software. This research process, applied to the Baptistery of Florence as a case study, seeks to define an operational methodology for modeling cultural heritage by providing some critical ideas for future studies.

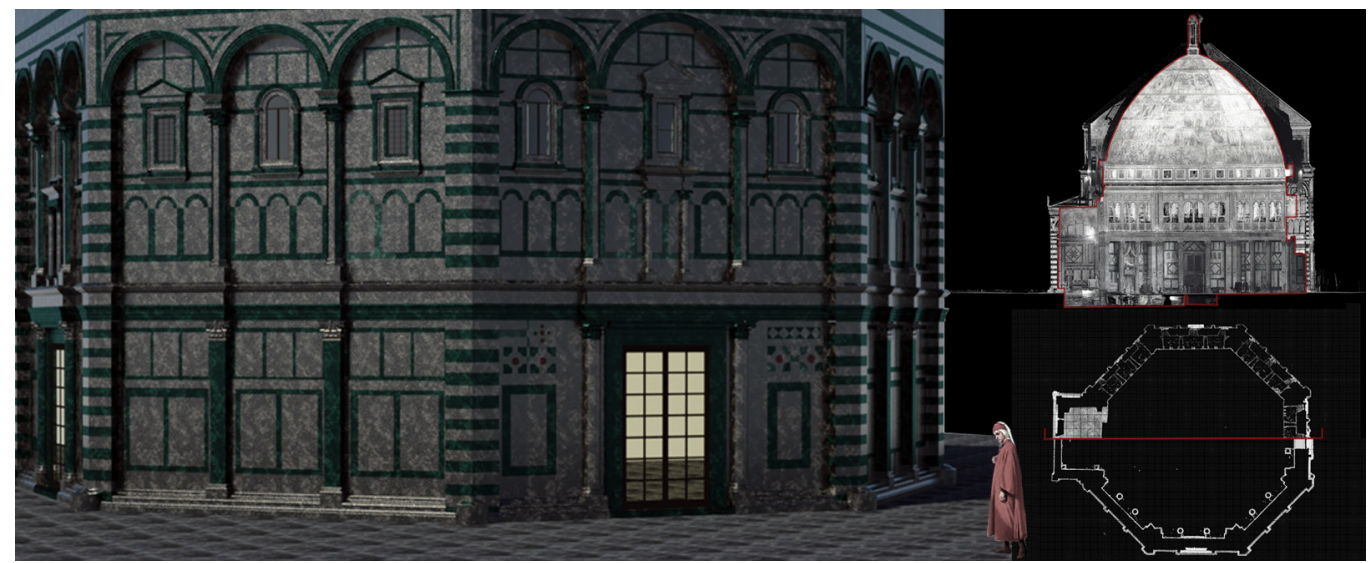




\section{Introduction}

The potential of the so-called Heritage-BIM or H-BIM systems is undeniable; however, the theoretical and operational questions relating to the rigidity of modeling tools remain an unresolved problem when we involve elements of cultural heritage, where in fact high flexibility is required.

By their nature, 3D models are made up of geo-referenced units, relating primarily to geometry, topology, materials, etc; at the same time, the digital storage technologies, analysis and management of information have found in the three-dimensional models the substrate on which to develop their potential [Centofanti et al. 20 I 6]. BIM offers the possibility to manage an important quantity of heterogeneous data which, going beyond the simple geometric aspect, can constitute a digital archive of data and information, useful for the documentation and conservation of historical architecture (fig. I). The translation of the complexity of the historical building into a digital three-dimensional model produces important questions on the procedures to be adopted. Parametric and informative modeling of monuments is much more difficult, both in terms of geometric transposition of the continuity of the real world, and of its qualitative and semantic description, compared to modeling from scratch. The BIM approach to a historic building differs primarily in the quantity and type of starting data. In the cultural heritage environment, there is a large amount of data available to be analysed and selected; it is necessary to establish how to catalogue them, encode them and store them within the model (or models) to allow maximum data interoperability and the creation of a coherent BIM model. Making a good model facilitates reading and understanding of the building itself. The HBIM model also differs and above all in the architectural forms involved and the way of representing them within the model in terms of geometric and informative attributes.

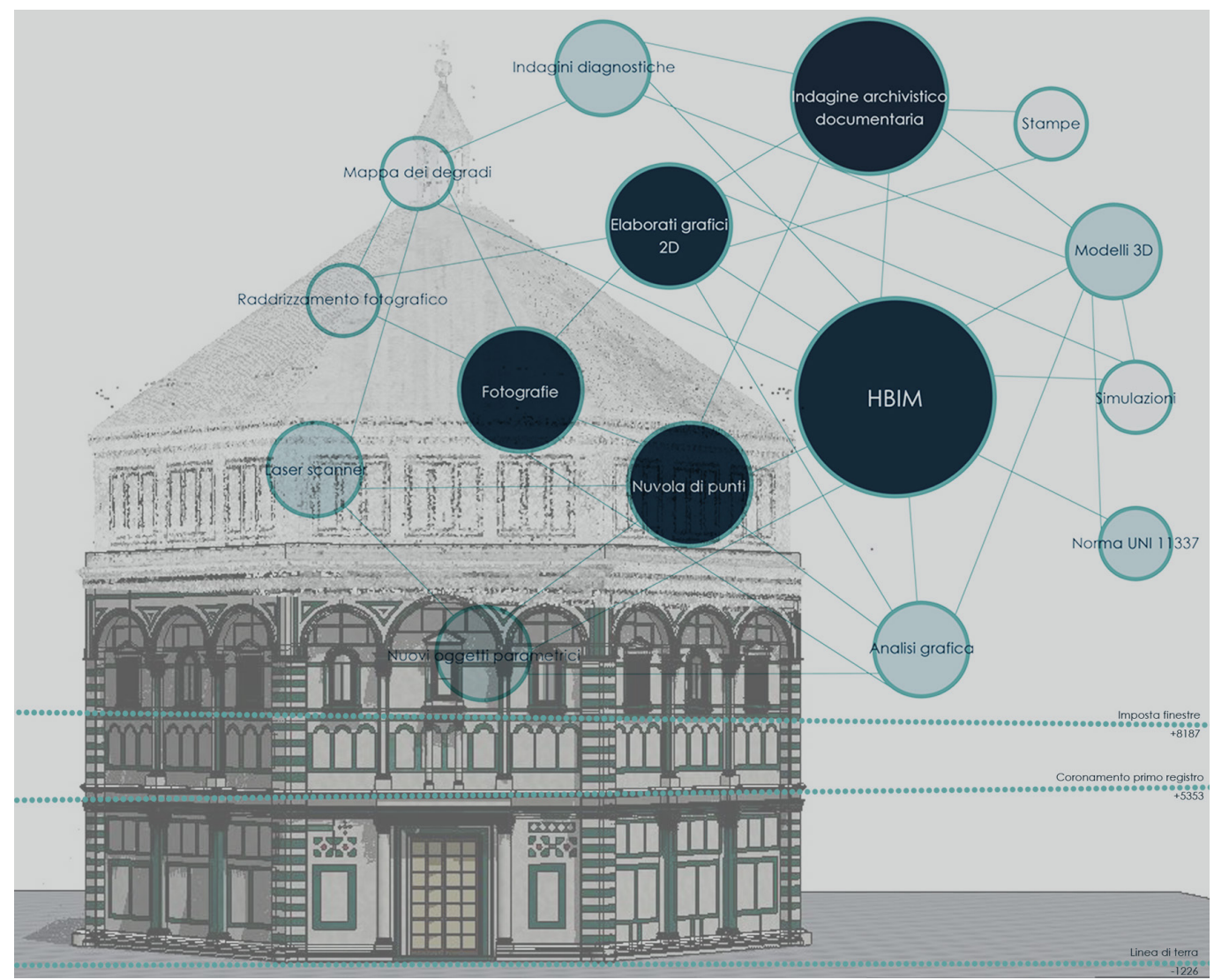


Fig. 2. Segmentation of the Baptistery of San Giovanni. Ortho-based photo processing.
Fig. 3. Hierarchical identification of the architectural elements. Ortho-based photo processing
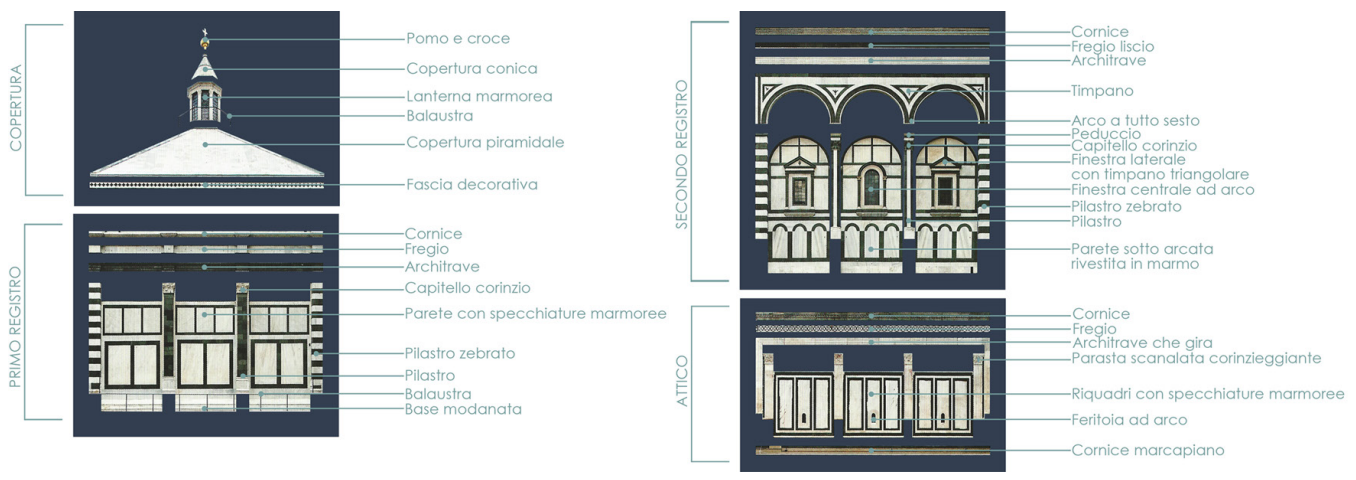

These difficulties are associated above all with the intrinsic rigidity of the parametric modeling workflow, subjected to 'libraries' of digital objects that clash with the variability and uniqueness of the built environment, especially when 'historical' or in a poor state of conservation. While the transition from numerical models to geometric models implies a critical operation of 'discretization', in the BIM construction, the model involves a further interpretative step: the semantic structuring of its compositional elements. This undeniably subjective ontological operation goes far beyond recognizing the geometric characteristics of the elements. In the BIM environment, in fact, the modeller proceeds from the continuum of reality to discrete digital by exploring a domain, additive and hierarchical, in which digital objects find their place. The aggregation of these objects, subject to reciprocal relationships of incorporation or belonging as well as constraints and interaction rules, guides the effective construction of the virtual information model.

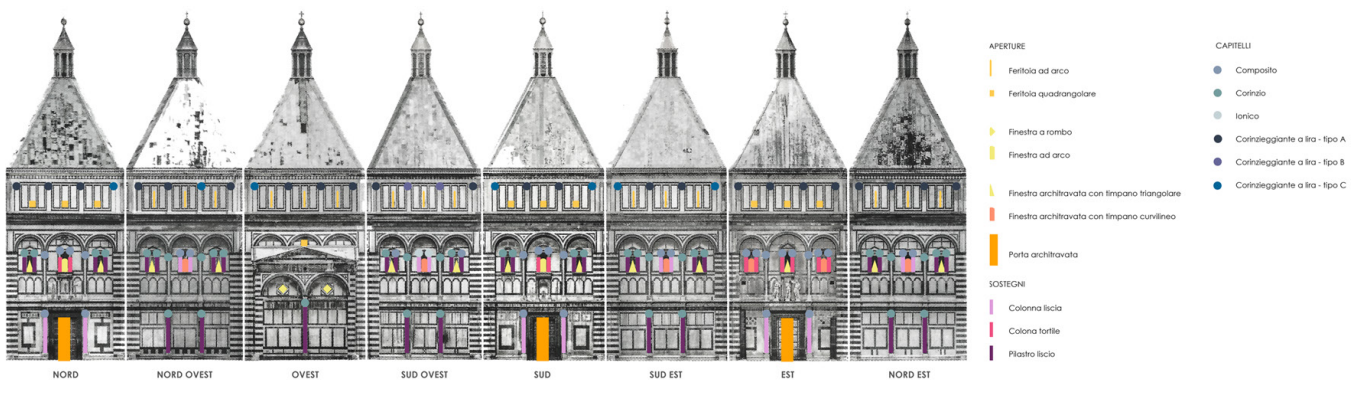

The term HBIM was coined by Murphy [Murphy, MC Govern, Pavia 2013] which presents it as an innovative solution, in which architectural elements are objects that integrate different information. About this, HBIM is an interscalar system, consisting in the modeling of architectural elements parameterized on the rules of the treaties and data deriving from investigations on the building [Campi et al. 20 I8]. The substrate of these models are point clouds, from TLS [I] and SfM [2], which affect the historical architectural organism. Numerous researches in this area have highlighted the potential and problems related to the definition of a workflow for the BIM modeling of objects. From the GreenSpider plug-in, for the creation of shapes [3], to the definition of specific shape grammars for individual types of construction elements (e.g. vaults), to the use of VPL (Visual Programming Language) software such as Autodesk Dynamo for the representation of generable elements using certain rules and parameters. There was also talk of the creation of nested parametric families, or through the hierarchical aggregation of the components according to the structural, functional and decorative function [4], and, for the BIM modeling of irregular and complex shapes, the 
Fig. 4. BIM modeling methodology applied to the cultural heritage (graphic elaboration by the author). import of NURBS to which they are added information about materials. Other researchers have reasoned on the topic of the semi-automatic conversion of the point cloud into semantic objects [Remondino et al. 20 18], however always highlighting how resorting to the BIM process for built heritage is rather expensive in terms of time and resources used. It is important, therefore, to understand up to which Level of Development one must actually go in modeling the basic construction elements, since the creation of discontinuous structural models in which the elements of the masonry (blocks or stone elements) and the joints of mortar are discretized individually, it is computationally expensive and difficult to manage [Currà et al. 2019].

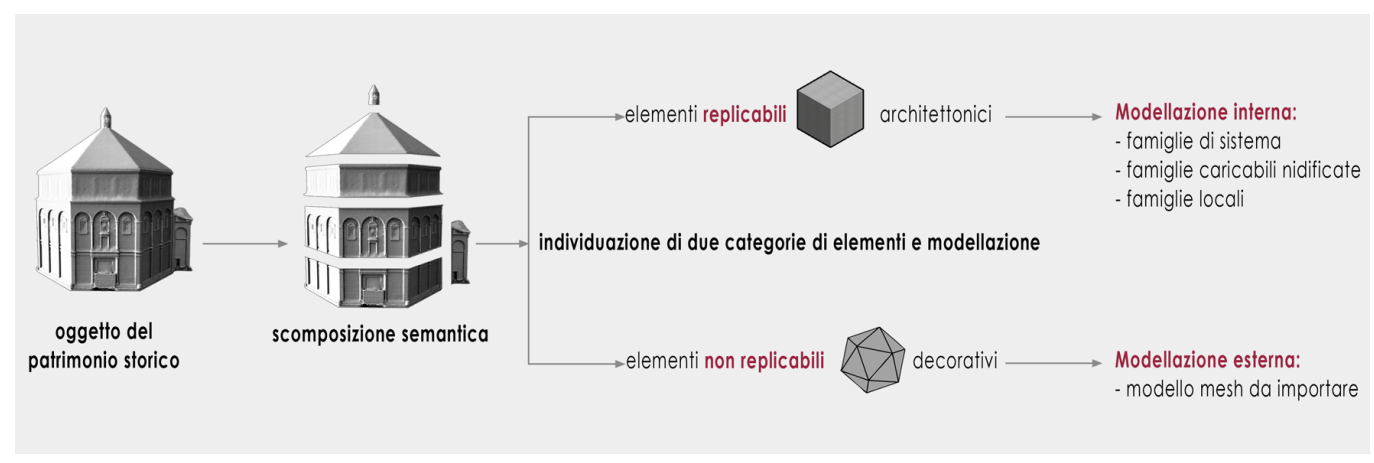

\section{Deep knowledge of the monument}

The aim of the research is to investigate the methods of construction of three-dimensional models through HBIM processes; the first step towards the knowledge of the historical artefact is undoubtedly the collection of historical documentation and archival research, which can provide essential indications about the construction phases of the building, the units of measurement used, the materials, the stratigraphies etc. The acquired data become a first base of support for BIM modeling, which is joined by the relevant data, which constitute a fundamental aspect for all activities concerning objects of the existing architectural heritage. Point clouds, from TLS to digital photogrammetry regarding the monument, offer an high amount of data that documents the existing condition of buildings and describes a specific phase of their life cycle. However, let's not forget that these acquisition methods, although they have become the standard in the practice of surveying, conserving and managing cultural heritage, stop at the 'skin' of the building. They ignore the building's stratigraphy, information necessary for the purpose of correct BIM modeling, and therefore to be integrated with further investigations, such as thermography, magnetometry etc. or simply with the data available from the historical documentation.

\section{From survey to HBIM: an application methodology}

Survey data constitute the starting point of the modelling; however it is necessary to articulate the methodological process in different preliminary phases. The study of a correct application method facilitates BIM modeling and in fact it is necessary especially for cultural heritage. In this specific application field, more than ever, it is necessary to start from the breakdown of the building into constitutive elements. Each artefact can be decomposed into a coordinated set of simple and complex elements, which together determine its structure. The analysis of these parts within a built architecture is defined, in a more or less appropriate way, semantic segmentation. The segmentation operation facilitates the study of historical monuments and integrates heterogeneous information and attributes, useful for 
describing and characterizing the object. We can describe the building as a collection of hierarchically organized elements, identified by a specific architectural vocabulary [Fallavolita et al. 20 I8]. Particular attention in this operation must be paid in analysing how these objects are assembled from a typological and morphological point of view since this problem occurs every time you approach the BIM modeling of an artefact: understanding how each element is constituted and how binds to others within the model.

The segmentation of historic buildings also requires an architectural vocabulary related to the knowledge of all those structural, functional and decorative components, such as mouldings, architectural orders, entablatures and profiles, and their geometric and topological characteristics, in terms of connections between the parts, in order to fully describe the monument (figs. 2, 3). The large amount of data and digital models produced brings out the need to catalogue and classify the data obtained through support tools of the interpretative phase. New modeling procedures and solutions and semantic annotation make segmentation operations easier and faster, according to different approaches such as colour similarity and spatial proximities, shape detection, distance between planar faces [Hichri et al. 20 I 3]. As already mentioned, when approaching a BIM model for historic buildings, we need to pay attention to the architectural forms involved and the way of representing them within the model as geometric attributes. In fact, we don't have to use traditional modelling rules, which allow the creation of complex geometries with loft operations, sweeps and particular extrusions. On the contrary, after defining which elements are to be modeled and how they are related to each other, it is necessary to define the modeling methods also on the basis of the information available. We recognized two macro categories of elements to be modeled: replicable elements and unique non-repeatable elements. The first category includes architectural and construction elements, such as types of walls, floors, vaults, columns, capitals, pillars, windows and entablatures; the second includes the sculptural and stone elements, furnishings and all those elements that constitute a unicum inside the building. Once we have recognized the distinctions between categories of elements, we will analyse which

Fig. 5. At the top, monument acquisition methods: terrestrial laser scannig and gigapixel images; below the survey drawn from historical archival research: plan and elevation and cut of the Temple of San Gio. Batista, engraving by Bernardo Sgrilli based on a design by Giovan Battista Nelli in Glovan Battista Nelli in Description and Studies dis. Maria del Fiore, di S. Maria del Fiore, Firenze 1733, plate $\mathrm{XVII}$, and Drawing by Corinth, excavations of 1895 in Pietramellara, C. Baptistery of San Giovanni in Florence, relief and critical study 1973.
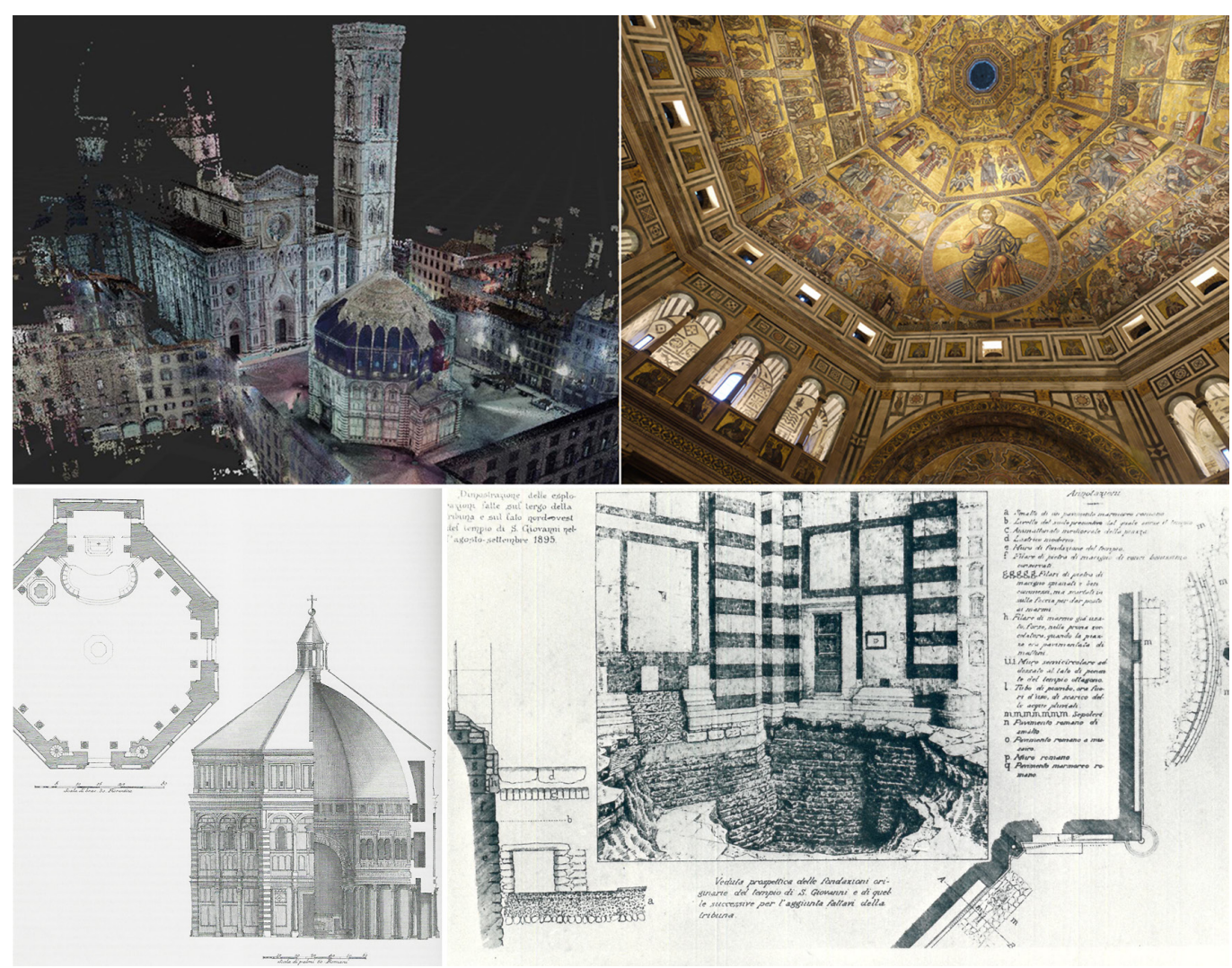


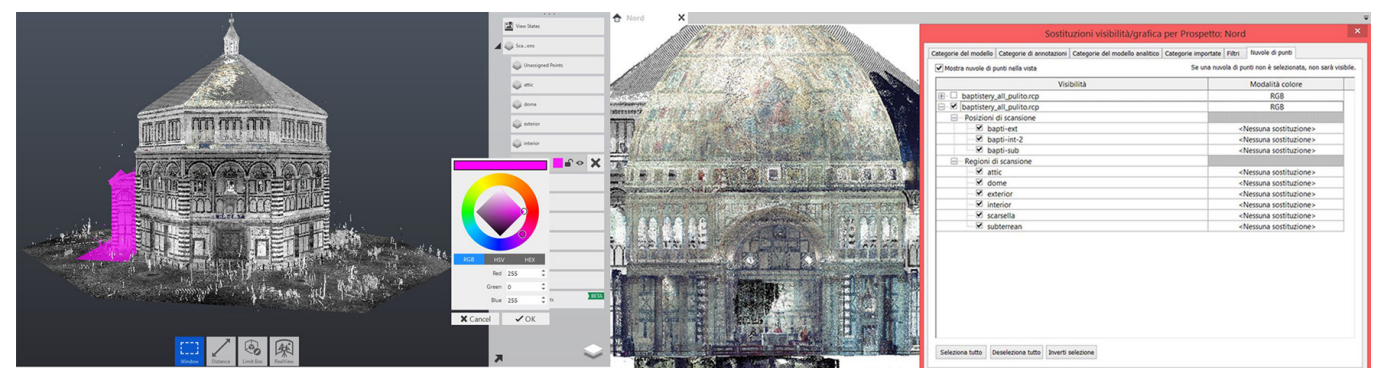

are the different modeling methods of the same. The replicable elements were all modeled within the BIM platform, using system families as regards the horizontal and vertical closing elements such as walls, floors and roofing, and nested loadable families or local modeling for all architectural elements. Within the software you have the possibility, for the structural parts, to model them directly through specific extrusion commands or, a convenient solution when you have less regular geometries as in the case of historical heritage, through the construction of a mass to which we can apply wall surfaces and floors, identifying the appropriate reference planes.

As regards the second category of objects, non-replicable elements characterized by complex geometries, such as the sculptural elements above the doors or the interior furnishings, we proceed by creating ad hoc mesh models on a point cloud or SFM models; in both cases, the models obtained must be imported subsequently into the BIM platform (fig. 4). However, this import operation will not be easy since the BIM platform used allows the import of only CAD formats, and it will be necessary more than one-step.

\section{Application to the case study: the Baptistery of San Giovanni in Florence}

The Florentine Baptistery, or 'll Bel San Giovanni' as Dante Alighieri called it [5], is a regular octagonal masonry construction, interrupted on the south side by the quadrangular apse, called scarsella. The dome has an octagonal plan covering, invisible from the outside by means of a pyramid-shaped structure with flat layers covered with white marble slabs. From a structural point of view, two levels can be recognized in height: one from the height of the floor to the soffit of the matroneo, and the other from the extrados of the matroneo to the gallery [Bartoli et al. 2017]. The analysis of the construction phases of the monument is preparatory to understanding this, as well as the ability to insert this information within the BIM platform for each element modeled through the use of 'phase filters'. Three main phases have been recognized for the construction of the artefact: a first phase, at the end
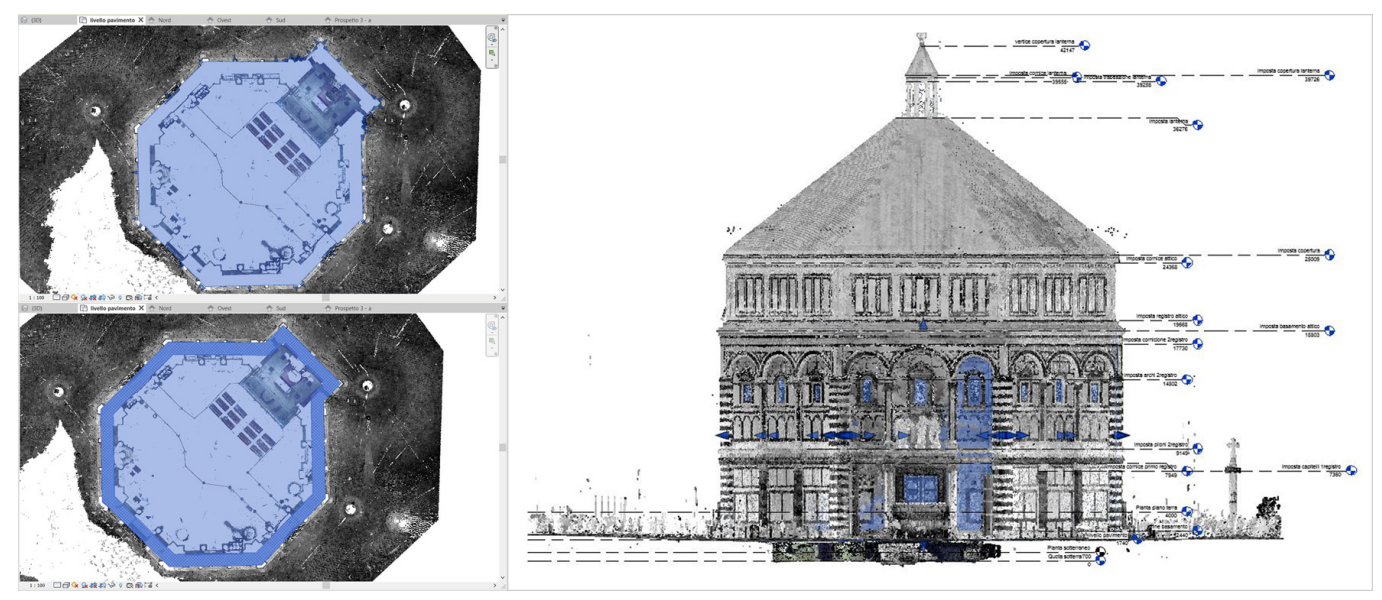
of the I Ith century, with the construction of the octagonal structure, a second phase, dated between the end of the II th century and the beginning of the I2th, with the construction of the marble cladding of the first two external registers and the construction of the lantern, and a last phase, dated to the first half of the thirteenth century, which sees the complete cladding of the covering surfaces and the galleries with the gold-based mosaic and the construction of the scarsella [Paolucci 1994]. The acquisition campaign was made by integrating different non-contact methodologies:TLS, SfM, infrared thermographic investigations, acquisition of gigapixel images (fig. 6). The data was integrated and managed within the Viscore CISA3[6] point cloud rendering software. A total of 62 individual TLS scans were performed, and aligned to create an overall point cloud with a half billion 3D data points acquired. The point clouds acquired by Faro laser scanners were recorded and aligned within the Viscore software, from which it was possible to obtain a series of high resolution snapshots which were preparatory to the modeling of the detailed elements; from this we then moved into Autodesk Recap to facilitate the import of the cloud into Revit.

A fundamental operation within Recap was to group the scans, dividing them by acquisition of the exterior, interior and underground, and above all the creation of specific scan regions (fig. 6) for the dome, the different registers and the scarsella. This facilitated modeling on the point cloud in Revit, as the BIM platform in the cloud import phase also allows the import of any segmentations made. Once the point cloud was imported, we proceeded with the geolocation of the project and the definition of the orientation, aligning the north of the project with the real north and thus generating views and elevations in line with the definitions used to date. We then proceeded with the setting of reference planes, essential not only to generate plan or section views, but also above all to constrain the modeled objects to specific planes or alignments of the architecture itself.

It was decided to start modeling the building envelope as a mass, as already anticipated, directly re-drawing from the imported point cloud (fig. 7), and the modeling took place in successive building phases, proceeding by registers. In this phase everything is modeled directly on the basis of the point cloud. The building stratigraphy has been defined based on recent studies and structural analyses and which have been found in the bibliography [7]. We have applied an external $8 \mathrm{~cm}$ thick layer of marble and the individual cladding slabs were subsequently defined by applying the finishes in white Carrara and green marble of Prato. All

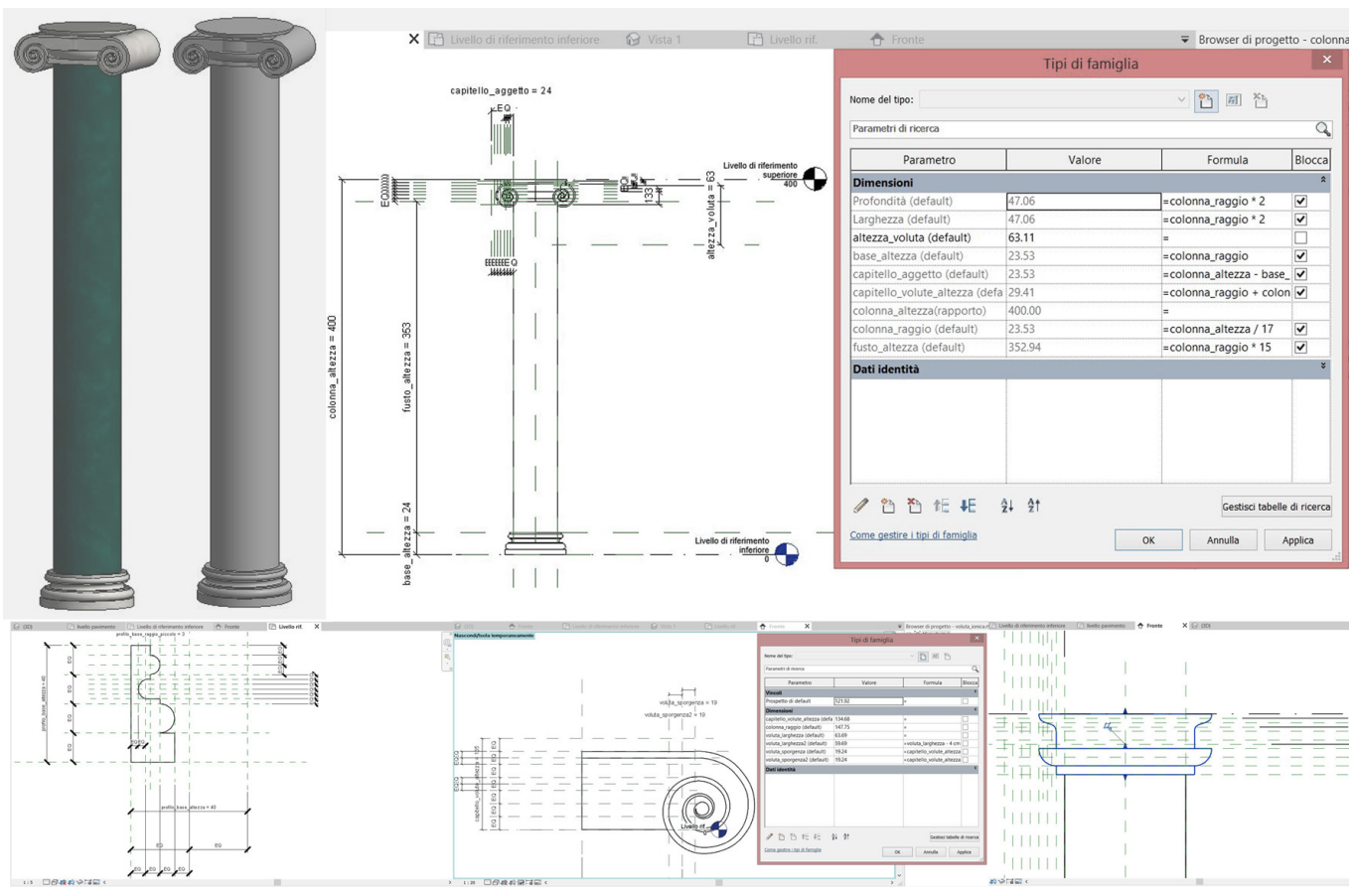


repeatable architectural elements, such as doors, windows, columns and trabeate nuts, have been treated as nested loadable families, or as elements modeled separately in the BIM platform and which are subsequently loaded within the project (fig. 8). These are more or less complex nested families; from those in which a profile family is used to obtain an extrusion on a track, to those in which both profile families and families of generic metric models are imported, for example for the construction of columns or windows (fig. 9). The profiles were modeled either directly in Revit parametrically or on a CAD basis and then imported. Like most parametric modeling software, Revit is also not able to extract data from the points of interest of the cloud in order to guarantee a faithful modeling of the geometries, but as with 2D rendering, even in the BIM modeling the point cloud is treated as a database, an image to be re-draw and from which to obtain the geometry of the building, and used as a metric trace. This process is clearly performed manually and consequently implies a strong degree of subjectivity in the interpretation and execution of modeling operations.

Fig. 9. Parametric modeling in plan and elevation of the nested family window gabled through profile families for the extrusion of the trabeations, the tympanum and the Windowsill and import of the nested composite pilaster family (on the right). The window family loaded and positioned of the project file (on the left).

Fig. 10. Application of the phase filter to the Corinthian pilaster element of the first register (on the left). Browser of the building phases entered and the materials used for the pilaster element (on the right).

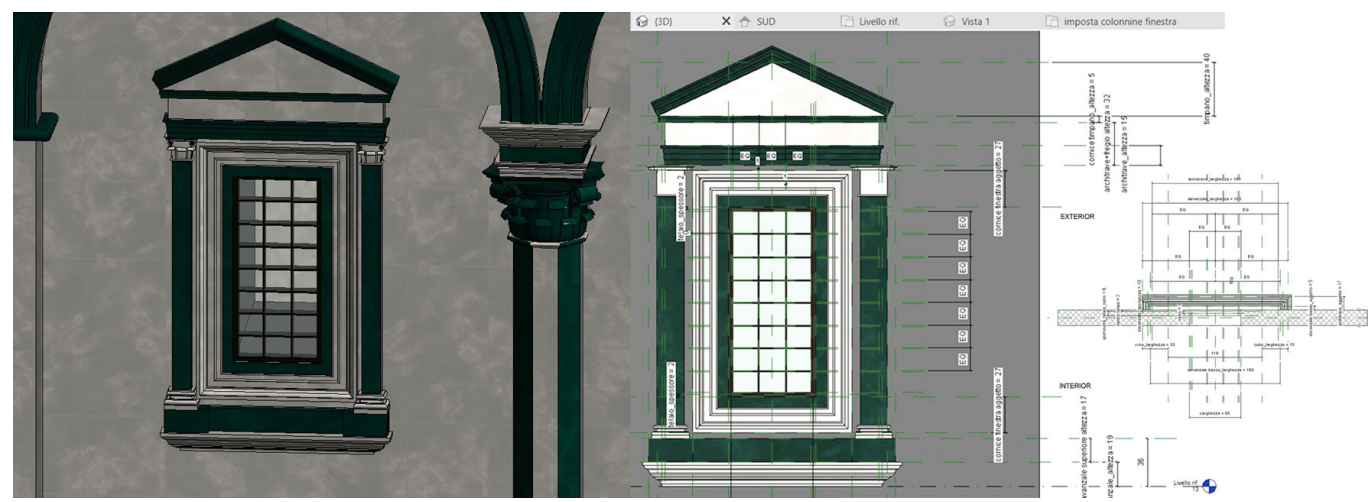

Finally, a further aspect of the model was investigated, namely that of the possibility of inserting element construction filters, the so-called phase filters. These allow us, in the modeling and management phase of the modeled element, to insert information about the creation phase of the element, providing information about the era of creation and to which building phase the monument belongs. The phase filters therefore give the possibility of generating an architectural model that also contains a synchronic analysis of the artefact, or that in its 'as-is' state it gives an account of the dating of some of its parts, visualizing them simultaneously. Obviously, the platform also makes it possible, as well as for the modeling of new buildings, to generate different views for each phase filter, thus giving the possibility of having a diachronic analysis, or of displaying separately the elements that belong separately to each construction phase.

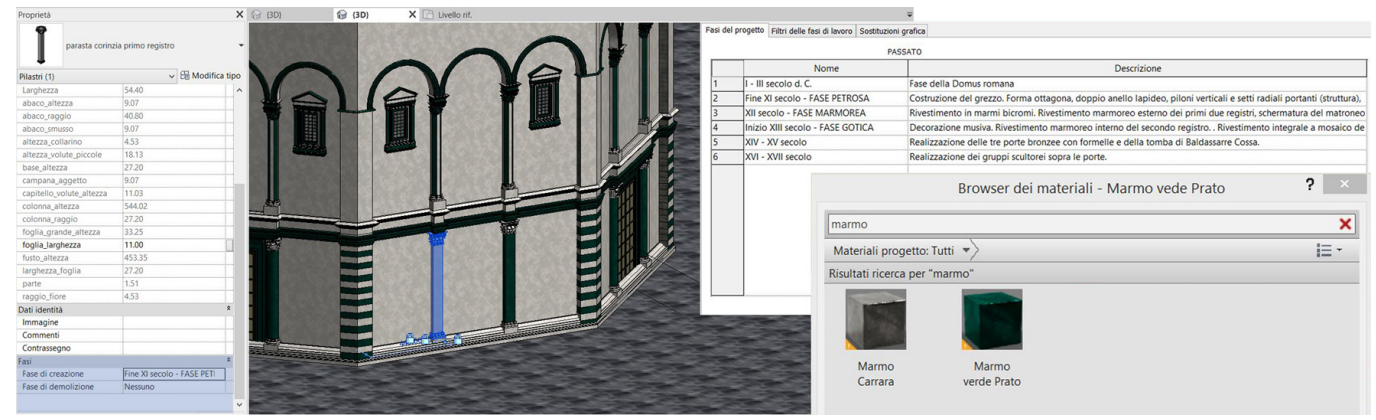




\section{Conclusions}

The research sought to investigate the different aspects of BIM modeling of a historical artefact, trying to define a possible operational process and highlighting some limits. When approaching the BIM modeling of a historical artefact, it is first necessary to clarify what the objectives are and above all the use of this model, to preventively define the level of details of this [Rossi, Palmieri 2018]. It is also clear that the LODs will never be homogeneous in the model, as they are affected by the real knowledge of the individual elements; for this purpose, the Level of Reliability of the elements should always be evident for each model [Bianchini, Nicastro 20 I8], in order to have always the control over the parts and allow their updates. The different representations that we can make within a BIM model of the LOR or of the different Levels of Detail or of the building phases to which each element belongs (Levels of Evolution), are themselves models, defined as 'thematic models' (fig . I0), chosen according to specific objectives.

Fig. I I. Redefinition of the BIM process data flow through the LODs and representation of the thematic levels. LOd of a pedestal (graphic elaboration by the author): LOR of the temple of Divo Claudio Attenn 2019]: LOE of the House 2019., LOE of [Bianchini, Nicastro 20 I8]

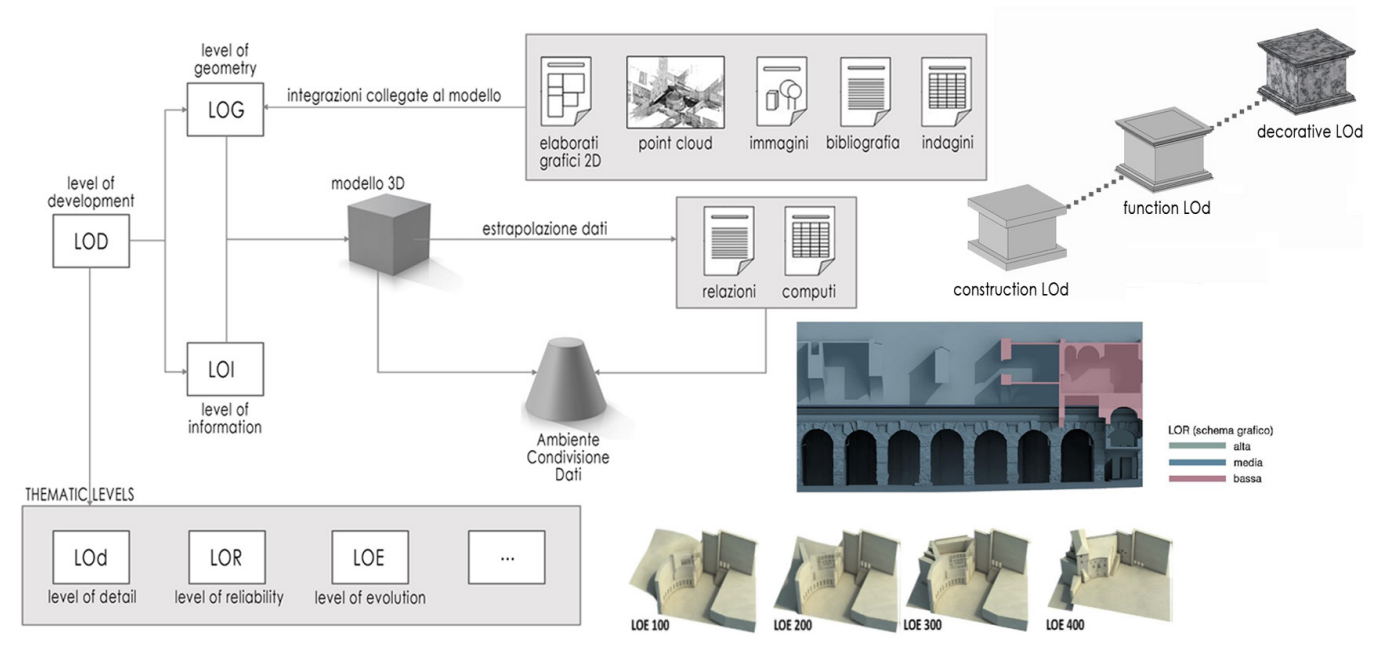

The growing use of these means of representation for architectural projects, engineering and now of restoration, requires us, as scholars, to find operational methodologies for application in the cultural environment without forgetting the aim that this modeling arises. If you look beyond the first obstacle, that is, the one for which BIM modeling was born for new buildings, for standardized elements, and try to define an operational strategy to apply it to cultural heritage, you realize that parametric modeling is no other than the representation of architecture as we have always recognized it. That is according to the Vitruvian concept of successive partitions and of the Architectural Order, and that the possibility of attaching to the model an big amount of information about materials, characteristics and all that we want to include within the property sheets, is a sensational opportunity for the field of restoration, management and representation of historical architecture. The BIM offers us, as scholars in the field of cultural heritage, the opportunity to collect for the first time all the information in our possession within a single platform (fig. I 2 ) in which it is also possible and above all to model the object. So why exclude this type of three-dimensional representation a priori and prefer another? However, every time you approach modeling it is always good to have clear the objectives that this project has. 


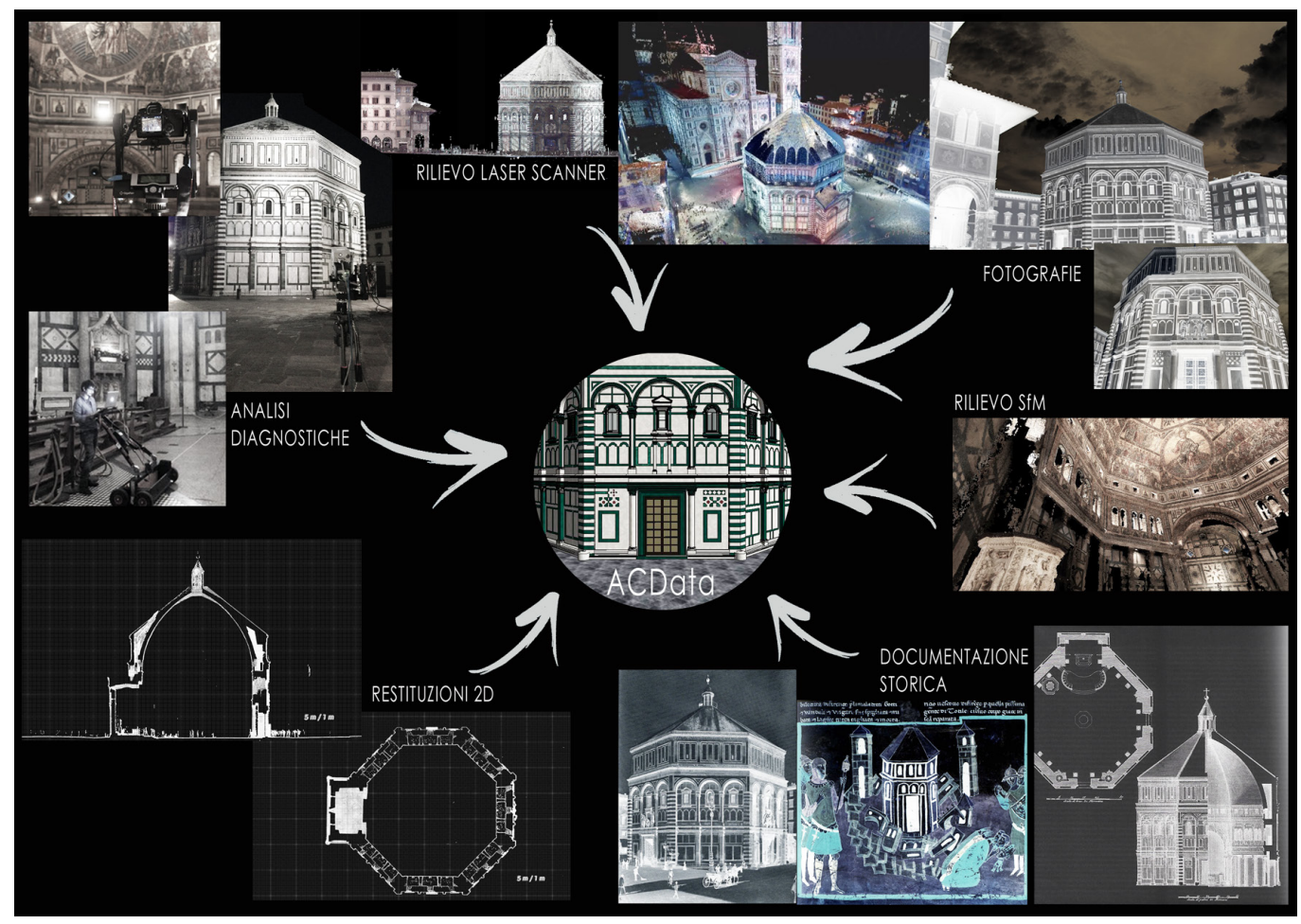

Notes

[I]Terrestrial Laser Scanner.

[2] Structure from Motion.

[3] "Non mi parean né ampi né maggiori che que' che son nel mio bel S. Giovanni, fatti per luogo di battezzatori", Dante Alighieri, Divina Commedia, Inferno, XIX, I6-18.

[4] Center of Interdisciplinary Science for Art, Architecture, and Archaeology della University of California San Diego.

[5] An internal and external finishing layer in $8 \mathrm{~cm}$ thick marble slabs characterizes the entire wall thickness; the internal core is characterized by a double ring masonry where the total thickness is $180 \mathrm{~cm}$.

\section{References}

Attenni Martina (2019). L'architettura romana al tempo del BIM. In Empler Tommaso, Fusinetti Alexandra (a cura di). 3D Modeling \& BIM. Modelli e soluzioni per la digitalizzazione. Roma: dei.

Bartoli Gianni, Betti Michele, Monchetti Silvia (2017). Modellazione numerica ed analisi strutturale del Battistero di San Giovanni a Firenze. In Gurrieri Francesco. Il battistero di San Giovanni: conoscenza, diagnostica, conservazione. Atti del convegno internazionale Firenze, 24-25 novembre 20 I4. Firenze: Mandragora, pp. I 35- 157.

Bianchini Carlo, Nicastro Saverio (2018). La definizione del Level of Reliability: un contributo alla trasparenza dei processi historic-BIM. In Empler Tommaso, Fusinetti Alexandra (a cura di). 3D modeling \& BIM, applicazioni e possibili futuri sviluppi. Roma: dei, pp. 209-225.

Bianchini Carlo, Nicastro Saverio (20 I8). From BIM to HBIM. In 2018 3rd Digital Heritage International Congress (Digital Heritage) held jointly with 2018 24th International Conference on Virtual Systems \& Multimedia (VSMM 20 I8).

Campi Massimiliano, Cera Valeria, Farella Elisa et al. (20।8). Un catalogo semantico per la conoscenza e la ricostruzione del paesaggio incompiuto. II caso di San Leucio. In Capano Francesca, Pascariello Maria Ines,Visone Massimo (a cura di). Delli aspetti de paesi. Vecchi e nuovi media per l'immagine del paesaggio. Napoli: FedOA, vol. 2, pp. 229-237.

Carnevali Laura, Lanfranchi Fabio, Russo Michele (2019). Built Information Modeling for the 3D Reconstruction of Modern Railway Stations. In Heritage an Open Access Journal by MDPI (2), pp. 2298-23I 0.

Centofanti Mario, Brusaporci Stefano, Maiezza Pamela (2016). Tra "HistoricalBIM" ed "HeritageBIM": Building Information Modeling per la documentazione dei beni architettonici. In Parrinello Sandro, Besana Daniela. Reuso 20 I6. IV Convegno Internazionale sulla documentazione, conservazione e recupero del patrimonio architettonico e per la tutela paesaggistica. Firenze 6-8 ottobre 2016. Firenze: Edifir, pp. 42-5I. 
Currà Edoardo, D'amico Alessandro, Angelosanti Marco (2019). HBIM per la conoscenza e la rappresentazione della costruzione storica. II caso di villa Palma - Guazzaroni a Terni. In Garda Emilia, Mele Caterina, Piantanida Paolo. Colloqui. AT. e Ingegno e costruzione nell'epoca della complessità. Atti del congresso Torino, 25-27 settembre 20 I 9. Torino: Politecnico di Torino, pp. 628-637.

Fallavolita Federico, Ballabeni Massimo, Foschi Riccardo, et al (2015). Semantic description of the three-dimensional models of Bologna porches. In SCIRES.IT, Vol 5, Issue I, pp. $31-40$.

Garagnani Simone (2013). Building Information Modeling and real world knowledge: A methodological approach to accurate semantic documentation for the built environment. In Digit 20 I 3. Fed I9th Int'IVSMM, I Oth Eurographics GCH, 2nd UNESCO Mem World Conf Plus Spec Sess fromCAA, pp. 489-496.

Hichri Nourredine, Stefani Chiara, De Luca Livio, et al (2013). Review of the "as-built BIM" approaches. In The International Archives of the Photogrammetry, Remote Sensing and Spatial Information Sciences. Proceedings of the 3DARCH 20I3. Trento, 25-26 February 20 I3. Göttingen: Copernicus GmbH, vol. XL-5/WI, pp. I07-I I 2

Murphy Maurice, MC Govern Eugene, Pavia Sara (2013). Historic Building Information Modelling - Adding intelligence to laser and image based surveys of European classical architecture. In ISPRS Journal of Photogrammetry and Remote Sensing, n. 76, pp. 89- 102.

Oreni Daniela, Brumana Raffaella, Georgopoulos Andrea et al. (2013). Hbim For Conservation And Management Of Built Heritage: Towards A Library OfVaults And Wooden Bean Floors. In ISPRS Annals of the Photogrammetry, Remote Sensing and Spatial Information Sciences, Volume II-5/WI, 20I3 -XXIV International CIPA Symposium, 2-6 September 20I 3, Strasbourg, pp. 215-22|.

Paolucci Antonio (1994). Il battistero di San Giovanni a Firenze. Firenze: Cosimo Panini Editore.

Remondino Fabio, Grilli Eleonora, Petrucci Giulio (20 I 8). Supervised segmentation of 3D cultural heritage. San Francisco: IEEE.

Rossi Adriana, Palmieri Umberto (2019). LOD per il patrimonio architettonico: la modellazione BIM per la fabbrica di Solimene. In Disègno, n. 4, Giugno 2019.

\section{Author}

Giorgia Potestà, La Sapienza Università di Roma, giorgia.potesta@uniromal.it

To cite this chapter. Potestà Giorgia (2020). Conoscenza e rappresentazione del patrimonio storico costruito in ambiente BIM. Criticità e possibili metodologie applicative/Knowledge and representation of Cultural Heritage in a BIM environment Critical issues and possible application methodologies. In Arena A., Arena M., Brandolino R.G., Colistra D., Ginex G., Mediati D., Nucifora S., Raffa P. (a cura di). Connettere. Un disegno per annodare e tessere. Atti del $42^{\circ}$ Convegno Internazionale dei Docenti delle Discipline della Rappresentazione/Connecting. Drawing for weaving relationships. Proceedings of the 42th International Conference of Representation Disciplines Teachers. Milano: FrancoAngeli, pp. I363-I 384. 